\title{
EFFECTS OF INITIAL ANISOTROPY ON THE FINITE STRAIN DEFORMATION BEHAVIOR OF GLASSY POLYMERS
}

\author{
Ellen M. Arruda, ${ }^{*}$ Mary C. Boyce, and Harald Quintus-Bosz $\dagger$ \\ The Massachusetts Institute of Technology
}

(Communicated by Kenneth Neale, Université de Sherbrooke)

\begin{abstract}
Solid phase deformation processing of glassy polymers produces highly anisotropic polymer components as a result of the massive reorientation of molecular chains during the large strain forming operation. Indeed, the polymer preform used as the starting material is usually anisotropic owing to its prior deformation history. The process end product has often been fashioned for a particular application, i.e. to possess an increased flow strength along a particular axis, thereby exploiting the orientation induced anisotropy effects. The fully three-dimensional issues involved in the use of glassy polymer components include anisotropic flow strengths, limiting extensibilities, and deformation patterns. These characteristics have been altered by the initial forming operation but are obviously not expected to be enhanced in all directions. The presence of anisotropy in structural components may also lead to premature failure or unexpected shear localization. In this report the effects of initial deformation and the associated anisotropies are investigated through uniaxial compression tests on preoriented polycarbonate (PC) and polymethylmethacrylate (PMMA) specimens. The evolving anisotropy is monitored by testing materials preoriented by various amounts of strain and under different states of deformation. The tensorial nature of the anisotropic material is characterized by examining the preoriented material response in three orthogonal directions. A model for the large strain deformation response of glassy polymers has been shown by ARRUDA and BOYCE [in press] to be well predictive of the evolution of anisotropy during deformation in initially isotropic materials. Here the authors evaluate the ability of the model developed in ARRUDA and BoYCE [in press] to predict several aspects of the anisotropic response of preoriented materials. Using material properties determined from the characterization of the isotropic material response and a knowledge of the anisotropic state of the preoriented material, model simulations are shown to accurately capture all aspects of the large strain anisotropic response including flow strengths, strain hardening characteristics, cross-sectional deformation patterns, and limiting extensibilities. Although anisotropy has been shown to evolve with temperature and strain rate in BOYCE, ARRUDA and JAYACHANDRAN [in press] and also state of deformation in ARRUDA and BOYCE [in press], we submit an experimental observation that the subsequent deformation response of preoriented polymers may be predicted using only a measure of optical anisotropy, and not the prior strain or thermal history. Optical anisotropy, as measured for example by birefringence, therefore represents a true internal variable indicative of the evolution of anisotropy with inelastic strain, state of strain, and temperature.
\end{abstract}

\section{INTRODUCTION}

Polymer components are often manufactured by any of a number of processes such as forging, stamping, or extruding, known collectively as solid phase deformation processes. In such processing operations a glassy polymer preform is subjected to large strains while exposed to spatially and time varying temperatures, pressures, and strain rates. The preform is usually anisotropic in the as-received condition because of the roll-

*Now at The University of Michigan, Department of Mechanical Engineering and Applied Mechanics, Ann Arbor, Michigan.

†Currently at XRE Corporation, Littleton, Massachusetts. 
ing or extruding associated with the initial forming of solid polymer sheet or bar stock. Processing of an anisotropic material under the conditions described above for solid phase deformation must not only account for the direction-dependent properties of the anisotropic polymer preform but should also anticipate undesirable features of anisotropic behavior, such as premature fracture and undesirable shear banding. The component produced from a solid phase deformation processing operation is invariably anisotropic, exhibiting direction-dependent flow strengths, limiting extensibilities, deformation patterns, and strain hardening characteristics. Whereas assessment of short-term performance may simply require testing of the anisotropic nature of the polymer end product, design of processing operations to predict dimensional stability, meet net shape specifications, and address structural integrity considerations requires accurate, three dimensional characterization of the effects of anisotropy on the behavior of glassy polymers during finite straining.

Previous experimental work on preoriented polymers includes studies of the effect of preorientation on the elastic constants and yield in glassy polymers by BROWN, DUCKETT, and WARD [1968], BROWN and WARD [1968], RAWSON and RIDER [1971], RIDER and Hargreaves [1969] and by Berg, Sun, and Magill [1989]. Rider and Hargreaves [1969] oriented PVC by hot rolling then tested in uniaxial tension at various angles to the rolling direction. They found the onset of yielding to occur in localized shear bands when testing off-axis from a principal orientation direction. Studies on oriented PET tested in tension by BROWN and WARD [1968] describe observations of deformation bands in terms of the structure of oriented polymers. For oriented PET sheet in which the material was tested in tension and in simple shear at different angles to the draw direction, shear localizations were found by BROWN, DUCKETT, and WARD [1968] to always occur upon off-axis tensile testing but not always in shear. WARD [1984] measured the change in natural draw ratio as a function of preorientation in polyethylene terephthalate (PET) where he found the draw ratio of the material to reduce with increasing axial preorientation. In the shear band studies of BROWN, DUCKETT, and WARD [1968], BRoWN and WARD [1968], and of RAWSON and RIDER [1971], the strong Bauschinger effect exhibited by these materials has been modelled by a scalar parameter that monitors the accumulation of an internal stress, this scalar parameter was used in a modified form of the anisotropic yield criterion of HILL [1950]. The buildup of an internal stored stress has been experimentally verified by ADAMS and FARRIS [1988] using a deformation calorimeter and also by the calorimetry and DSC experiments of OLEYNIK [1990].

Lee and coworkers have modelled both the tensile behavior and the solid phase cup forming of polycarbonate using an elastic-viscoplastic constitutive relationship in VEST, AMOEDO, and LEE [1987] and in LEE and LUKEN [1986]. Their models use as the strain hardening element the Mooney-Rivlin rubber elasticity formulation that has been shown by ARRUDA and BOYCE [1993] to not be capable of capturing the three dimensionality of the strain hardening response of rubber materials at large strains. LEE and LUKEN [1986] similarly find this representation not suitable for capturing strain hardening in glassy PC. ARRUDA and BoyCE [in press] have recently modelled the large strain response of glassy PC and PMMA, using physical bases for initial yield and strain hardening responses. Their model accurately captures the three dimensional nature of the strong state of deformation dependence on the strain hardening response of these materials with a new rubber elasticity element shown in ARRUDA and BOYCE [1993] to also capture strain hardening in rubbery materials. 
The present study of the effects of initial anisotropy in glassy polymers concerns investigation of the three dimensional yield and postyield characteristics of preoriented materials. Highly anisotropic materials were produced by an initial orientation process, and both the magnitude of initial strain and the deformation process used to preorient the materials were varied. Several specimens were cut from each preoriented sample to allow for testing of the anisotropic response by uniaxial compression in three directions. The preoriented specimens varied strongly in the amounts of strain endured prior to failure (limiting extensibilities), flow strengths, deformation patterns, and strain hardening characteristics.

The three dimensional nature of the anisotropy in these materials warrants characterization in terms of a tensorial representation of the stored internal stress rather than a scalar parameter used in earlier studies of BROWN and WARD [1968], RAWSON and RIDER [1971] and of RIDER and HaRgreaves [1969]. A previous study by ARRUDA and BOYCE [in press] on initially isotropic glassy polymers showed the evolution of anisotropy with state of deformation and magnitude of strain to be the result of three dimensional orientation of the polymer molecular network in response to plastic straining. Birefringence has been identified as a morphological descriptor of the oriented polymer, capable of a measurable amount of evolution with both magnitude and state of plastic strain. The orientation induced anisotropy has been modelled in the previous work as an internally stored backstress tensor that correctly accounts for the strong state of deformation dependence exhibited by two initially isotropic glassy polymers, PC and PMMA.

It is desirable to determine whether the newly developed model of orientation induced anisotropy in initially isotropic materials has accurately captured the three dimensional aspects of the polymer response, and therefore represents a true constitutive relationship of glassy polymer deformation. Using material properties identified for the isotropic polymer in the previous study, and a knowledge of the orientation state in the anisotropic specimen, uniaxial compression simulations are performed on anisotropic polymers. Comparisons are made of the flow strengths, extensibilities, cross-sectional deformation patterns, and strain hardening characteristics exhibited by the experimental results and predicted in the simulations.

\section{EXPERIMENTS}

\section{II.1. Compression testing procedures}

Constant strain rate compression tests were performed on PC and PMMA using an Instron 1350 servohydraulic testing machine controlled by a Macintosh personal computer. Details of the testing apparatus are given in ARRUDA and Boyce [in press]. Initially isotropic PC and PMMA specimens were deformed to various amounts of strain in either uniaxial compression or plane-strain compression. Uniaxial compression specimens were cubes measuring $12.7 \mathrm{~mm}$ on edge. Plane-strain specimens measured $9.5 \mathrm{~mm} \times 9.5 \mathrm{~mm} \times 12.7 \mathrm{~mm}$. The plane-strain compression test consisted of a hardened tool steel die that housed the polymer specimen, constraining flow in one horizontal direction while compressing vertically. See Fig. 1 for a schematic of the plane-strain compression die and specimen geometry. One test result is reported for uniaxial compression at $75^{\circ} \mathrm{C}$, which utilized a Research Inc. Model E4 radiant furnace attached around the Instron load train; the remainder of the tests reported here were conducted at room temperature. 


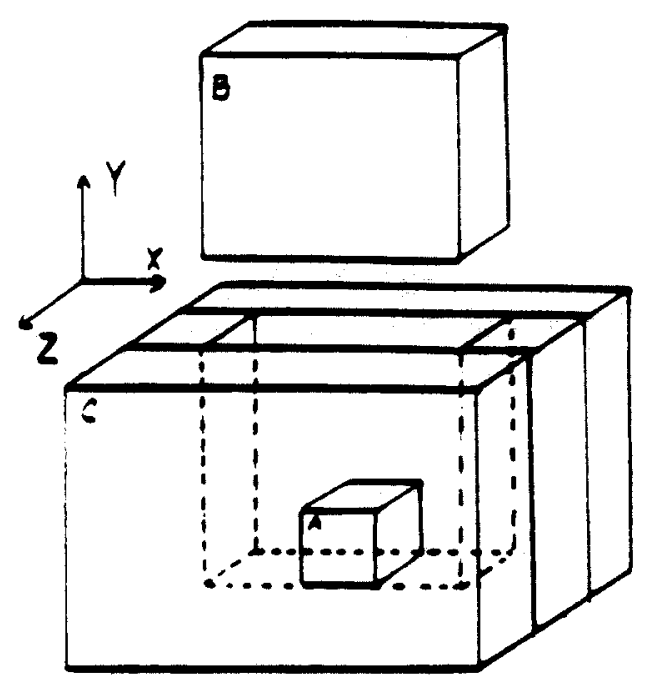

Fig. 1. Sketch of plane-strain compression testing apparatus. Specimen (A) is compressed by punch (B) in the $\mathrm{Y}$ direction and expands in the $\mathrm{X}$ direction. The die $(\mathrm{C})$ constrains the specimen along the $\mathrm{Z}$ axis.

The anisotropic materials that resulted from either preorientation process described above were cut into cubes for retesting using a Buehler Isomet low speed saw equipped with a silicon carbide wafer blade. Care was taken to ensure the cutting operation produced no additional deformation sufficient to affect the material response. The isomet cutting operation was evaluated based on birefringence measurements of isotropic materials that were sliced using this same procedure. Because the cutting operation did not impart a birefringence to the isotropic material, the operation was considered not to affect the deformation response of the preoriented specimens.

All tests on preoriented specimens were conducted in uniaxial compression; the compression direction, state of initial orientation, and magnitude of initial orientation were variables in this study. The current strain in the preoriented material was measured at the time of recompression. The expected amount of extensibility was approximated for each individual preoriented specimen based on the magnitude and direction of the major orientation axis with respect to the subsequent compression axis, and each specimen was deformed to that estimate of its limiting strain. In this way, the full range of the anisotropic response of the material could be captured during the recompression tests.

Recompression testing also included some interrupted tests for which the deforming cross-section of the anisotropic specimens could be measured as a function of plastic strain. All three specimen directions were measured with a micrometer to give the current plastic strain with respect to the initial anisotropy in the material along the compression axis, and the cross-section dimensions.

\section{II.2. Birefringence measurements}

Birefringence measurements were conducted using a Leitz microscope to transmit polarized light through the thin polymer slices of deformed specimens. A Berek S-N 3311 compensator provided a measure of the specimen retardation of light in nanometers 
using the known optics of the compensator as described in GRAHAM [c1970]. Birefringence relates to retardation in the following manner:

$$
\Delta \eta=\frac{\Gamma}{t}
$$

where $\Delta \eta$ is the birefringence (or relative retardation), $\Gamma$ is the retardation in nanometers calculated from the compensator reading, and $t$ is the specimen thickness.

\section{MODELLING}

\section{III.1. Model constituents}

The companion paper to this work by ARRUDA and BoYCE [in press] detailed a three dimensional representation of the highly anisotropic response of initially isotropic $\mathrm{PC}$ and PMMA. This anisotropy is particularly evident in the strong state of deformationdependent strain hardening response of these materials. The authors showed previously how the model developed therein predicted the deformation response of both materials in plane-strain compression based entirely on material properties determined from the uniaxial compression deformation response. This earlier work is the first successful prediction known to the authors of the large strain response of any material in a given deformation state based on the properties determined from characterization of a different deformation state.

The three dimensional model of the rate, temperature, pressure, and state of deformation-dependent response of these materials, described in detail in ARRUDA and BoyCE [in press], is used here to further assess its ability to characterize polymer anisotropy. This model monitors two barriers to continued inelastic deformation, an 'isotropic' barrier and an 'anisotropic' barrier. The 'isotropic' barrier to chain segment rotation proposed by ARGON [1973] is shown to respond to the current deformation rate, temperature, and pressure. The Argon model of initial yield in glassy polymers gives the strain rate at which inelastic deformation ensues once the applied shear stress reaches the isotropic barrier to chain segment rotation,

$$
\dot{\gamma}^{P}=\dot{\gamma}_{0} \exp \left[-\frac{A s}{k \theta}\left\{1-\left(\frac{\tau}{s}\right)^{5 / 6}\right\}\right]
$$

In eqn $2, \dot{\gamma}_{0}$ is the preexponential factor proportional to the attempt frequency described by HASAN, BoYCE, LI, and BERKo [1993], $s \equiv 0.077 \mu /(1-\nu)$ is the athermal shear strength, $\mu$ is the elastic shear modulus, $A s$ is the zero stress level activation energy, $\tau$ is the effective equivalent shear stress, $k$ is Boltzmann's constant, and $\theta$ is absolute temperature. The stress at which inelastic strain occurs, $\tau$, depends on temperature and strain rate in the Argon equation. Boyce, PARKs, and Argon [1988] have added the pressure dependence of yield in glassy polymers to the model and have taken the athermal resistance to evolve with inelastic strain that phenomenologically captures the evolution of this isotropic barrier to continued flow during strain softening. HASAN, BOYCE, LI, and BERKo [1993] have recently reported a model for the strain softening regime that relies on the physical aging characteristics of the materials. 
A second barrier to continued inelastic straining evolves highly anisotropically with temperature, strain, and state of deformation. This 'anisotropic' barrier is due to the orientation of the molecular chain network within the glassy polymer during inelastic straining and results in extensive strain hardening beginning at strains in excess of $30 \%$ in the isotropic polymer. Characterization of strain hardening requires a three dimensional representation of molecular network orientation. The anisotropic barrier to chain alignment has been modelled using the rubber elastic deformation model of ARRUDA and BoYCE [1993], shown to predict the state of deformation dependence in rubber materials. This barrier is termed the backstress; the principal components of the deviatoric backstress tensor are given by

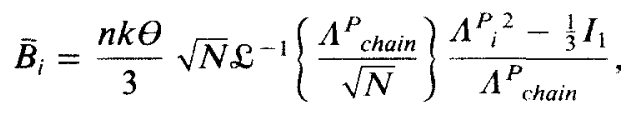

where $I_{1}=\left(A_{1}^{P^{2}}+\Lambda_{2}^{P^{2}}+{\Lambda^{P}}^{2}\right)$ is the first principal invariant of the stretches. The $A^{P}{ }_{\text {chain }}=(1 / \sqrt{3}) I_{1}{ }^{1 / 2}$ denotes the plastic stretch on a chain and the $A^{P_{i}}$ are the principal applied plastic stretches. In eqn $3, n$ is the chain density, $\mathcal{L}^{-1}\left(\Lambda^{P}\right.$ chain $\left./ \sqrt{N}\right\}$ is the inverse Langevin function, and $N$ is a statistical parameter related to the limiting value of chain stretch. The Langevin function is defined by:

$$
\mathscr{L}(\beta)=\operatorname{coth} \beta-\frac{1}{\beta}, \quad \beta=\mathscr{L}^{-1}\left\{\frac{\Lambda_{\text {chain }}^{p^{\prime}}}{\sqrt{N}}\right\} .
$$

This functionality will result in asymptotically increasing stress as the chain stretch reaches its limiting value, $\sqrt{N}$.

Tests on preoriented materials allow for a distinction between the contributions of each barrier to inelastic straining. The material is shown to possess memory by acknowledging the prior strain softened yield strength that corresponds to the current pressure, temperature, and strain rate. This material state is monitored by the isotropic barrier to inelastic flow. Initial yield is also strongly influenced by the stored backstress developed during initial deformation, the backstress enhances the compressive yield strength normal to a direction of prior orientation but reduces the compression flow stress normal to a direction of prior compressive deformation. Anisotropic strain hardening is found to respond to the conditions present at the time of the initial deformation; the important processing conditions during preorientation include the temperature, magnitude of strain, and state of deformation. The backstress is a monitor of the anisotropic resistance to inelastic flow caused by molecular chain network orientation.

\section{III.2. Mathematical formulation of constitutive model}

Details of the kinematics of finite strain plasticity used in modelling the isotropic glassy polymer are given in ARRUdA and BOYCE [in press]. See BoYCE, WEBER, and PARKs [1989] for a general discussion of the kinematics of large strain plasticity in crystals and glassy polymers. The various quantities involved are summarized below.

The deformation gradient, $\mathbf{F}$, is multiplicatively decomposed as described by LEE [1969] into elastic and plastic components

$$
\mathbf{F}=\mathbf{F}^{e} \mathbf{F}^{p}
$$


where $\mathbf{F}^{p}$ represents an unloaded, relaxed configuration obtained by elastically unloading without rotation, i.e. $\mathbf{F}^{e}=\mathbf{F}^{e T}$. The plastic component of $\mathbf{F}$ may be further decomposed into a symmetric stretch tensor $\mathbf{V}^{p}$ or $\mathbf{U}^{p}$, and an orthogonal rotation tensor $\mathbf{R}^{p}$ :

$$
\mathbf{F}^{p}=\mathbf{V}^{p} \mathbf{R}^{p}=\mathbf{R}^{p} \mathbf{U}^{p}
$$

The rate quantities include the velocity gradient, $\mathbf{L}$, and quantities arising from its decomposition

$$
\begin{gathered}
\mathbf{L}=\dot{\mathbf{F}} \mathbf{F}^{-1}=\mathbf{F}^{e} \mathbf{F}^{e-1}+\mathbf{F}^{e} \dot{\mathbf{F}}^{p} \mathbf{F}^{p-1} \mathbf{F}^{e-1} \\
\mathbf{L}^{p}=\dot{\mathbf{F}}^{p} \mathbf{F}^{p-1}=\mathbf{D}^{p}+\mathbf{W}^{p}
\end{gathered}
$$

where $\mathbf{D}^{p}$ is the plastic stretching tensor and $\mathbf{W}^{p}$ is the plastic spin. Without loss of generality (see for example BOYCE, WEBER, and PARKs [1989]) $\mathbf{W}^{p}$ is algebraically prescribed due to symmetry imposed on $\mathbf{F}^{e}$, while $\mathbf{D}^{p}$ remains to be constitutively prescribed. The magnitude of the plastic rate of shape change is prescribed by the Argon expression of eqn 2 and its direction is given by a normalized tensor, $\mathbf{N}$, in the direction of the deviatoric driving stress state, $\mathbf{T}^{* \prime}$.

$$
\begin{aligned}
\mathbf{D}^{p} & =\dot{\gamma}^{p} \mathbf{N} \\
\mathbf{N} & =\frac{1}{\sqrt{2 \tau}} \mathbf{T}^{* \prime}
\end{aligned}
$$

The effective equivalent shear stress is found from the tensorial difference between the total stress, or Cauchy stress, $\mathbf{T}$, and the convected back stress, $\mathbf{B}$

$$
\begin{aligned}
\tau & =\left[\frac{1}{2} \mathbf{T}^{* \prime} \cdot \mathbf{T}^{* \prime}\right]^{1 / 2} \\
\mathbf{T}^{*} & =T-\frac{1}{J} \mathbf{F}^{e} \mathbf{B} \mathbf{F}^{e T} .
\end{aligned}
$$

$\mathbf{T}^{*}$ represents that portion of the stress that continues to activate plastic flow. The deviatoric tensor of the driving stress state is denoted $\mathbf{T}^{* \prime 1}$. The elastic constitutive relationship gives the Cauchy stress as a function of the Hencky strain after ANAND [1979]

$$
\mathbf{T}=\frac{1}{J} \mathscr{L}\left[\ln \mathbf{V}^{e}\right]
$$

where $\mathbf{T}$ is the total stress, $\mathcal{L}$ is the fourth order tensor operator of elastic constants, $\ln \mathbf{V}^{e}$ is the Hencky strain, and $J=\operatorname{det} \mathbf{V}^{e}$. The portion of the total stress that is not dissipated in driving plastic flow is the stored backstress tensor, $\mathbf{B}$; the principal values of this deviatoric tensor are

\footnotetext{
${ }^{1}$ Note here that $\mathbf{D}^{p}$ is the plastic stretching in the relaxed configuration, yet we have made the approximation that its tensor direction, $\mathbf{N}$, is defined by the normalized $\mathbf{T}^{* \prime}$ direction, which is in the loaded configuration. Therefore this formalism neglects the small (approximately $5 \%$ since we have neglected the nonlinear viscoelastic contribution) elastic stretches in the direction of plastic straining.
} 


$$
\bar{B}_{i}=\frac{n k \theta}{3} \sqrt{N} \mathscr{L}^{-1}\left\{\frac{\Lambda_{\text {chain }}^{P_{\text {ain }}}}{\sqrt{N}}\right\} \frac{\Lambda_{i^{2}}^{{ }^{2}}-\frac{1}{3} I_{1}}{\Lambda_{\text {chain }}^{P}} .
$$

The principal values of the deviatoric backstress tensor are taken to be coaxial with the eigenvalues, $A^{P}$, of the left plastic stretch tensor, $\mathbf{V}^{p}$, and which are extracted as follows:

$$
\begin{aligned}
\mathbf{F}^{p} & =\mathbf{V}^{p} \mathbf{R}^{p} \\
\mathbf{V}^{p} & =\mathbf{Q}^{T} \Lambda^{P} \mathbf{Q} \\
\bar{B}_{i} & =\frac{n k \theta}{3} \sqrt{N} \mathscr{L}^{-1}\left\{\frac{\Lambda_{\text {chain }}^{P_{\text {a }}}}{\sqrt{N}}\right\} \frac{\Lambda_{i}^{P^{2}}-\frac{1}{3} I_{1}}{\Lambda_{\text {chain }}^{P}} \\
\mathbf{B} & =\mathbf{Q}^{T} \overline{\mathbf{B}} \mathbf{Q} .
\end{aligned}
$$

\section{III.3. Incorporating the initial anisotropy}

An initial plastic deformation gradient enters the formulation for anisotropic polymers, see for example BoYCE, PARKs, and ARgON [1989] for further discussion. The magnitude and state of strain resulting from the preorientation process are described by an initial deformation gradient $\mathbf{F}^{i}$. The relaxed configuration is achieved by elastically unloading, and the deformation gradient $\mathbf{F}$ is a product of the initial deformation gradient tensor and the additional gradient of the current deformation process, $\mathbf{F}^{p^{*}}$ :

$$
\begin{gathered}
\mathbf{F}=\mathbf{F}^{e} \mathbf{F}^{p} \\
\mathbf{F}^{p}=\mathbf{F}^{p^{*}} \mathbf{F}^{i} .
\end{gathered}
$$

The preorientation affects the total backstress tensor, the principal components of which depend on both the preorientation tensor and the reloading orientation. The backstress tensor of the total plastic deformation gradient in the relaxed configuration is coaxial with the left plastic stretch tensor,

$$
\mathbf{F}^{p}=\mathbf{V}^{p} \mathbf{R}^{p}
$$

\section{RESULTS}

\section{IV.1. Experimental results}

Results of uniaxial compression and plane-strain compression tests on isotropic PC are plotted in Fig. 2. The strong state of deformation dependence exhibited in Fig. 2 has been recently documented and explained by ARRUDA and BOYCE [in press] for PC and PMMA as being the result of a very anisotropic molecular orientation process that occurs in glassy polymers during plastic deformation. This evolution of anisotropy has been characterized in terms of the birefringence properties of oriented glassy polymers; birefringence measurements of ARRUDA and BoYCE [in press] confirmed that the three dimensional structures of specimens oriented under different states of deformation varied significantly with deformation state. Specifically, birefringence measurements estab- 


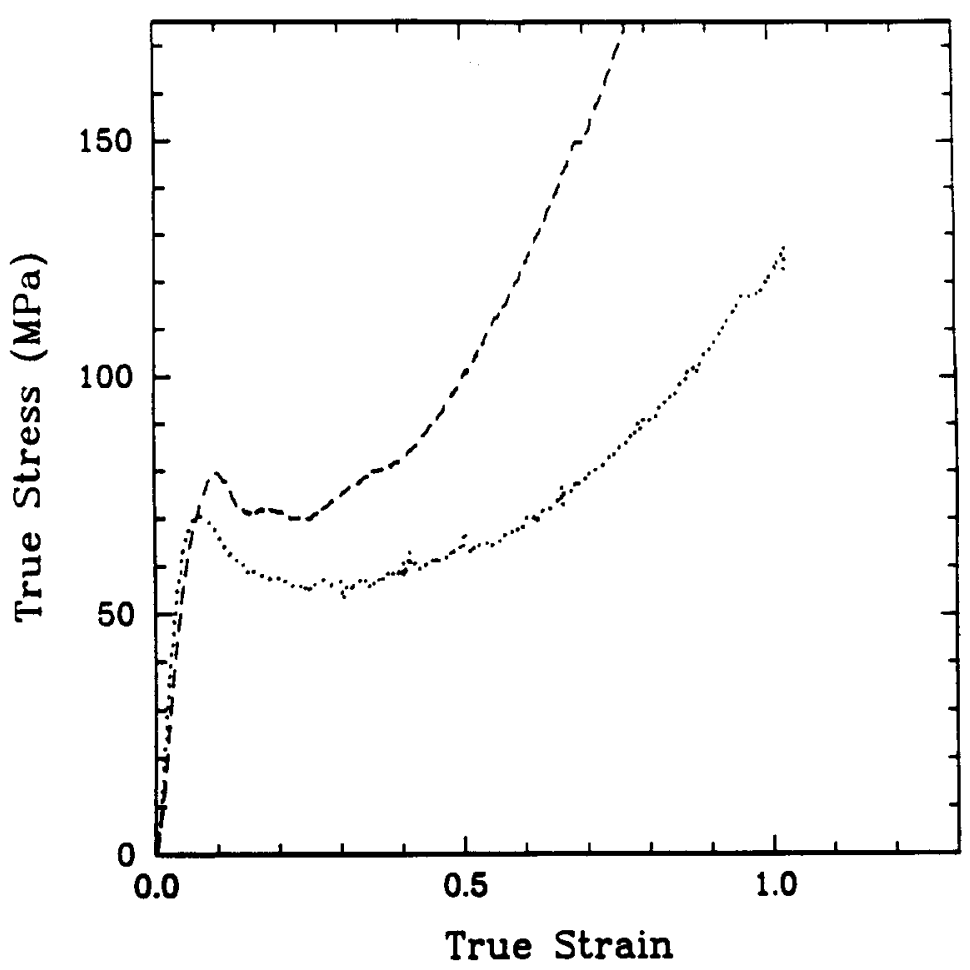

Fig. 2. Experimentally determined uniaxial compression and plane-strain compression stress-strain curves for $\mathrm{PC}$ at $\dot{\epsilon}=-0.001 / \mathrm{s}$ and room temperature.

lish that under uniaxial compression deformation the material develops axisymmetric anisotropy, whereas the state of orientation developed during plane-strain compression is fully three dimensional. Testing of materials preoriented by either uniaxial or planestrain compression with the constraint of a constant state of deformation during recompression serves to further illustrate and investigate the highly anisotropic nature of the oriented glassy polymer.

Figures 3 to 9 contain results of recompression tests on preoriented PC specimens. All recompression tests were conducted in uniaxial compression. Results include stressstrain responses and cross-sectional deformation maps for PC specimens initially loaded to various amounts of $\operatorname{strain}^{2}$ in either uniaxial or plane-strain compression, thereby providing a number of specimens with different initial states of orientation. Each figure of anisotropic stress-strain results includes the response to the initial deformation procedure used to preorient the material.

Table 1 contains the principal stretch state for each of the preoriented specimens and is provided to aid in the descriptions of the experimental results and comparisons with simulations. All results for preoriented materials will be discussed in terms of the convention established in this table. Chains are oriented along directions for which the stretch is $\lambda>1.0$ and coiled along directions having a stretch $\lambda<1.0$.

\footnotetext{
${ }^{2}$ This material recovers on the order of $25 \%$ of the total strain upon unloading and prior to redeforming the specimens. Strains reported in the following sections are the values of current plastic strain at the time of recompressing the materials unless otherwise noted.
} 


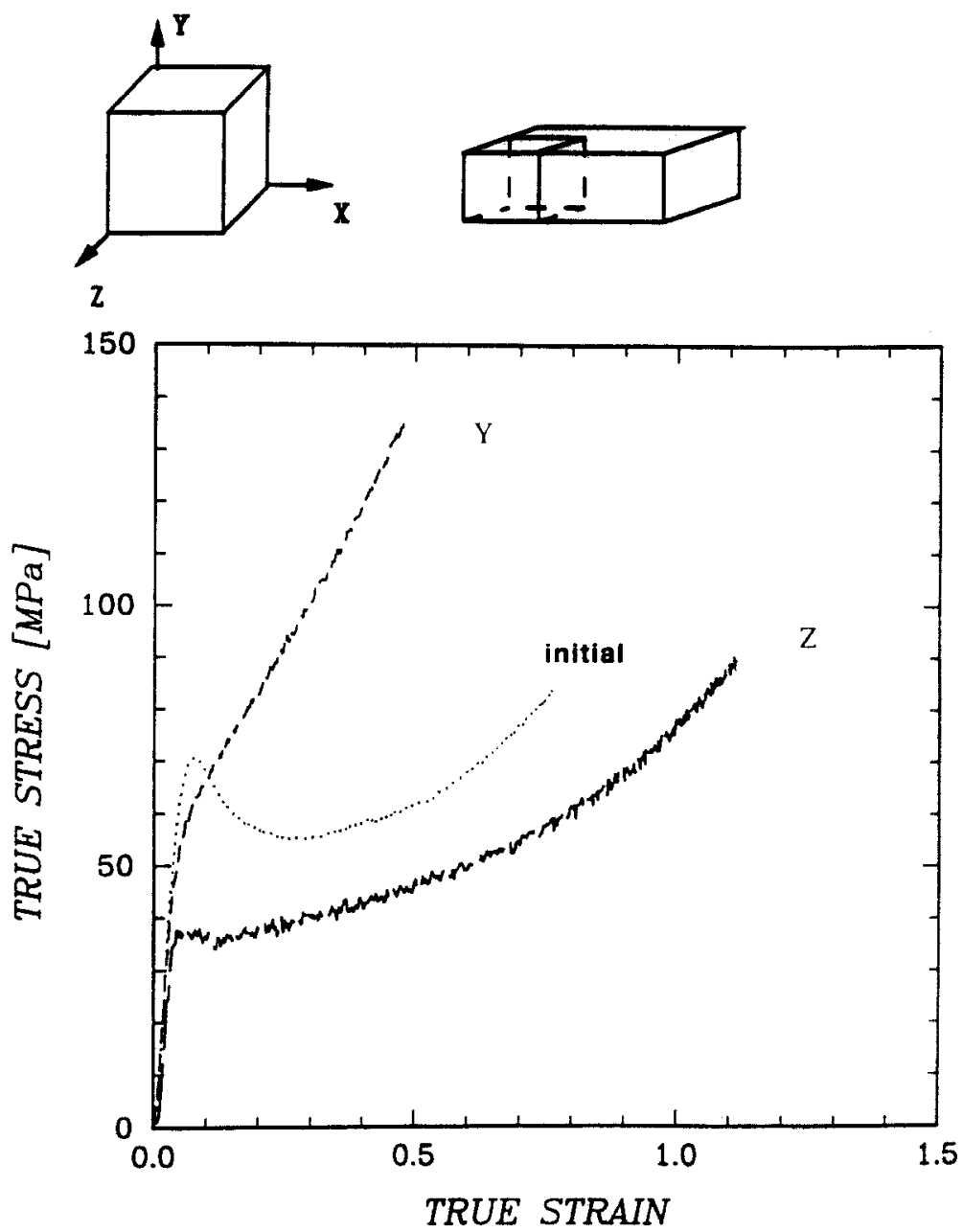

Fig. 3. Experimentally determined uniaxial compression stress-strain curves of the isotropic response of PC and anisotropic response as a result of transverse isotropy in PC prestrained by $\epsilon=-0.57$ at $\dot{\epsilon}=-0.001 / \mathrm{s}$ and room temperature.

Table 1. Summary of anisotropic cases considered in this article, including convention used to describe the directions in the preoriented materials

\begin{tabular}{lllll}
\hline Case & Recompression Axes & $\lambda_{X}$ & $\lambda_{Y}$ & $\lambda_{Z}$ \\
\hline $\begin{array}{l}\text { Uniaxial compression prestrain } \\
\text { (transverse isotropy) }\end{array}$ & & & & \\
Fig. 3 & $\mathrm{X}(=\mathrm{Z}), \mathrm{Y}$ & 1.3298 & 0.5655 & 1.3298 \\
Fig. 4 & $\mathrm{X}(=\mathrm{Z}), \mathrm{Y}$ & 1.4514 & 0.4747 & 1.4514 \\
Fig. 5 & $\mathrm{X}(=\mathrm{Z})$ & 1.2905 & 0.6005 & 1.2905 \\
Fig. 6 & $Y^{\prime}$ & 1.3962 & 0.5130 & 1.3962
\end{tabular}

the $\mathrm{Y}$ axis is rotated $45^{\circ} \mathrm{cw}$ about the $\mathrm{Z}$ axis to the $Y^{\prime}$ axis

Plane-strain prestrain

(three dimensional anisotropy)

Fig. 7

$X, Y, Z$

$X, Y, Z$

Fig. 8

$X, Y, Z$

1.6487

1.0000

Fig. 9

$1.6487 \quad 0.6065$

1.0000 

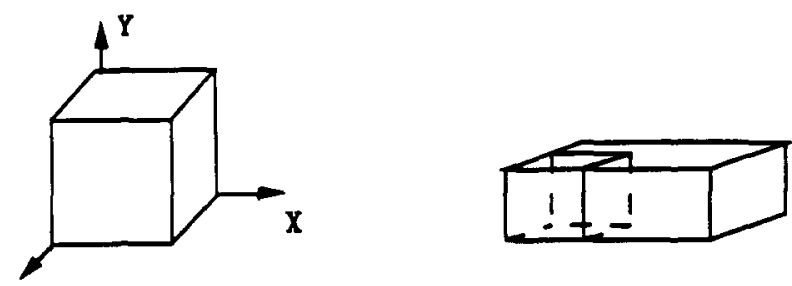

2

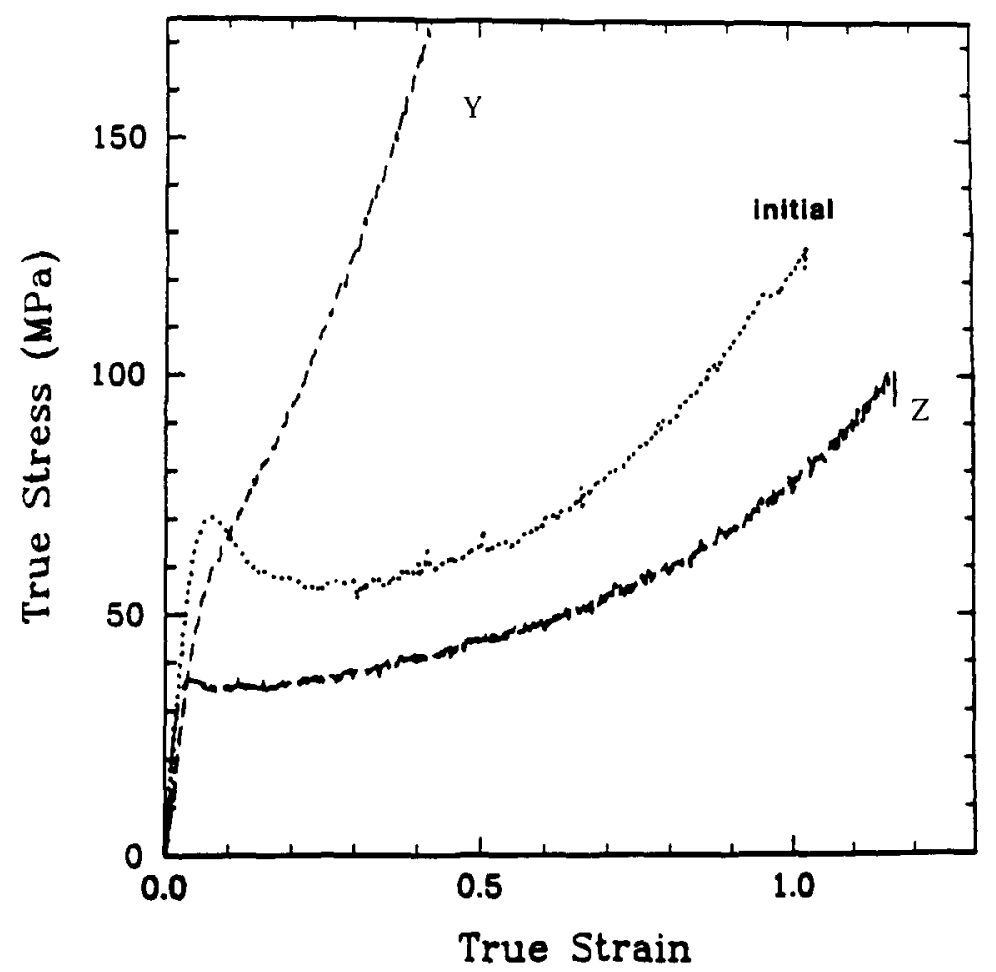

Fig. 4. Experimentally determined uniaxial compression stress-strain curves of the isotropic response of $P C$ and anisotropic response as a result of transverse isotropy in PC prestrained by $\epsilon=-0.75$ at $\dot{\epsilon}=-0.001 / \mathrm{s}$ and room temperature.

IV.1.1. Initial transversely isotropic orientation. Figure 3 shows the strongly anisotropic response of $\mathrm{PC}$ preoriented in uniaxial compression to a current plastic strain of $\epsilon=-0.57$. The specimen is biaxially oriented as a result of the initial compression; the material possesses transverse isotropy with respect to the original compression axis. Birefringence measurements of ARRUDA and BOYCE [in press] confirm the chains are preferentially oriented in the plane normal to the compression axis as a result of uniaxial compression. The data in Fig. 3 include the recompression responses along the original uniaxial compression direction (Y) and perpendicular to the initial compression axis (along the $\mathrm{Z}$ axis). The anisotropic effects include direction-dependent flow strengths, extensibilities, and strain hardening characteristics. Comparisons of the preoriented material responses with the isotropic behavior also included in Fig. 3 show that the anisotropic flow strength is increased for recompression normal to the plane of chain orientation and reduced for recompression along a direction of chain orientation. The 

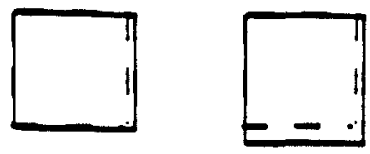

$.17 .2 \%$

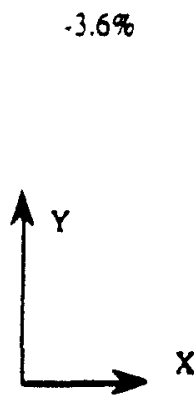

$-17.2 \%$

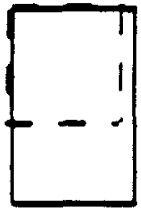

$-60.9 \%$

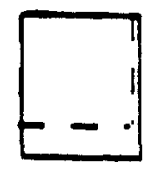

$.30 .0 \%$

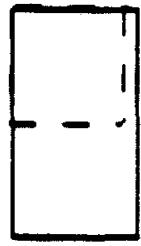

$80.4 \%$

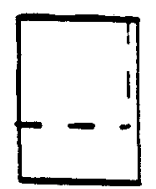

$43.9 \%$

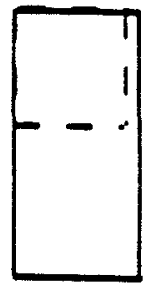

$.96 .3 \%$

Fig. 5. Experimentally determined deformation maps from micrometer measurements of the anisotropic evolving cross-section vs. plastic strain in anisotropic PC prestrained in uniaxial compression by $\epsilon=\cdots 0.5 \mathrm{I}$ at $\dot{\epsilon}=-0.001 / \mathrm{s}$ and room temperature.
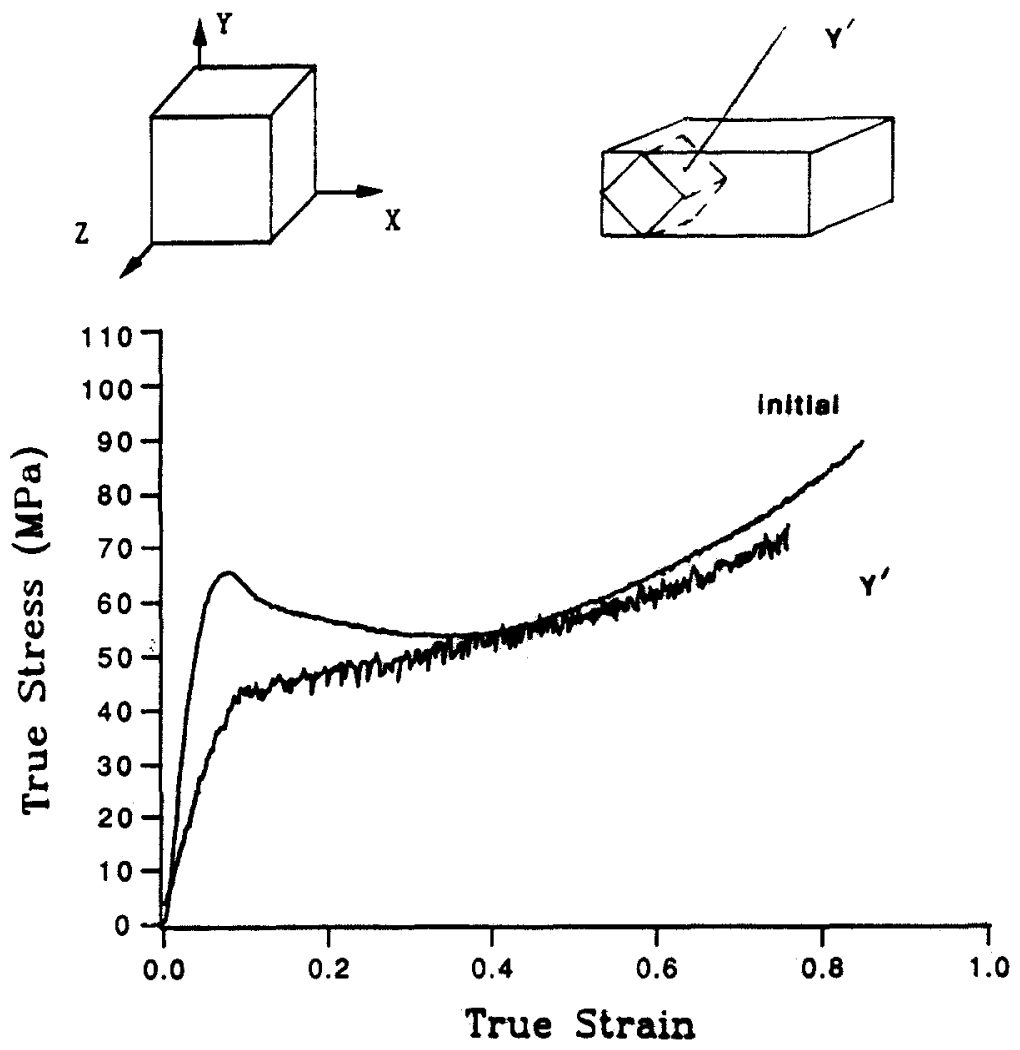

Fig. 6. Experimentally determined uniaxial compression stress-strain curves of the isotropic response of PC and anisotropic response as a result of principal chain alignment in a plane at $45^{\circ}$ to the recompression axis in $\mathrm{PC}$ prestrained by $\epsilon=-0.67$ at $\dot{\epsilon}=-0.001 / \mathrm{s}$ and room temperature. The anisotropic response included extensive specimen shear. 

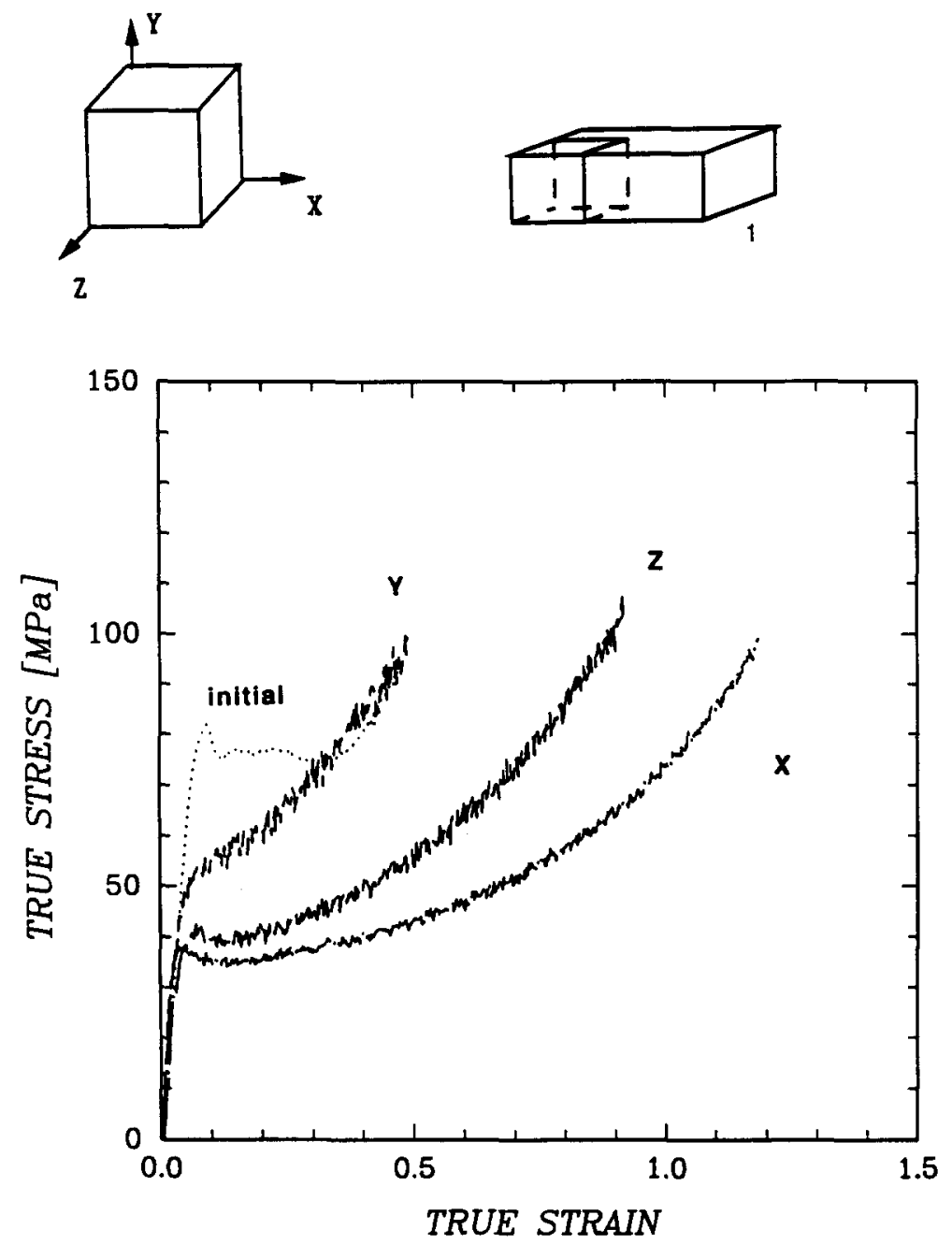

Fig. 7. Experimentally determined plane-strain compression stress-strain curves of the isotropic response of $\mathrm{PC}$ and anisotropic response as a result of three dimensional anisotropy in PC prestrained by $\epsilon=-0.35$ at $\dot{\epsilon}=-0.001 / \mathrm{s}$ and room temperature.

isotropic response includes a significant amount of strain softening from the initial yield stress level. Enhancing the yield strength of preoriented materials beyond the original isotropic value requires a significant amount of prestrain to orient the chains and combat the softening effect. The result of preorienting to small amounts of strain on the order of $20 \%$ to $35 \%$ is to actually decrease the flow stress in all directions.

It is perhaps useful to point out that the resistance to plastic flow in compression deformation experiments is always due to the presence or absence of preferential alignment of molecular chains in the plane normal to the current compression axis. The increased flow strength exhibited during recompression along the $Y$ axis is the response of the material continuing to align chains that had been partially oriented during the original compression. When the material is recompressed along the $\mathrm{Z}$ axis, the plane normal to the compression axis is the $\mathrm{XY}$ plane, which is in a state of anisotropic alignment; 

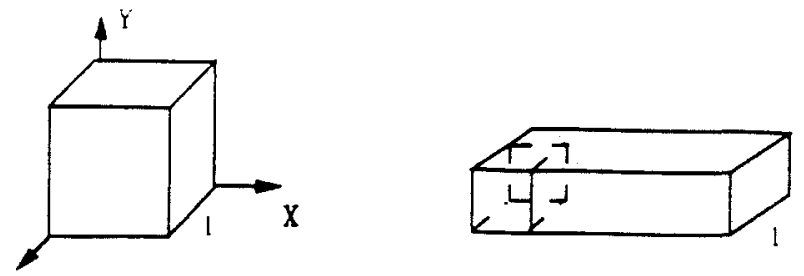

Z

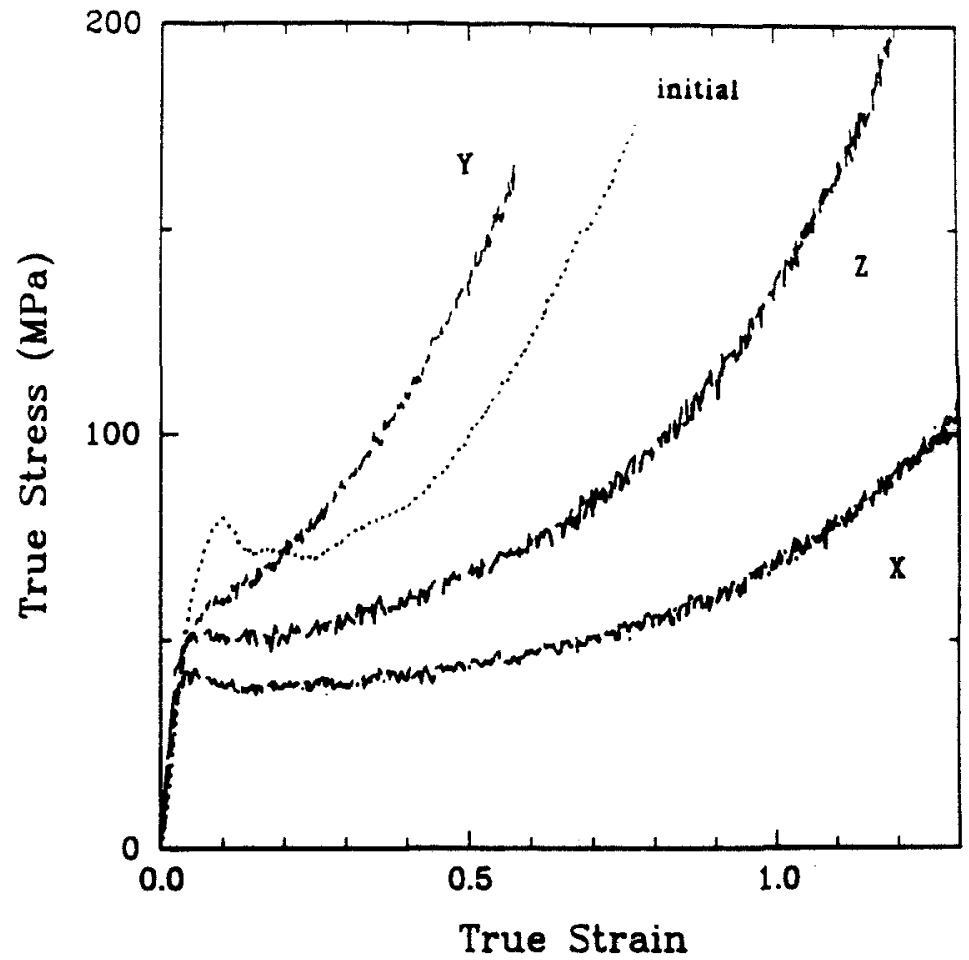

Fig. 8. Experimentally determined plane-strain compression stress-strain curves of the isotropic response of $\mathrm{PC}$ and anisotropic response as a result of three dimensional anisotropy in PC prestrained by $\epsilon=-0.50$ at $\dot{\epsilon}=-0.001 / \mathrm{s}$ and room temperature.

one principal direction is a direction of preferential alignment, and the other is the original compression axis in which chains are not stretched. The compression response in this case reflects the recoiling of the chains in the $\mathrm{Z}$ direction to a less oriented state. In addition, the resistance to flow in the XY plane is lower than the original isotropic resistance.

The other manifestations of the oriented molecular network include the postyield strain hardening characteristics and direction-dependent extensibilities. The extensibility of the material is greatly reduced for recompression along the $Y$ axis because such a procedure attempts to further orient chains in the $\mathrm{XZ}$ plane. The rapidly accumulating strain hardening response of the material during this process reflects a rapid approach to locking of the chains at the extensibility limit. In contrast, compression along the $Z$ axis recoils the oriented chains along the $Z$ direction while forcing alignment in the XY plane of the cross-section. The process of initially reversing the effects of the 
- $\gamma$ Recompression

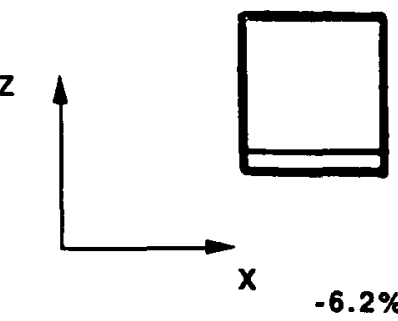

$-6.2 \%$

-X Recompression

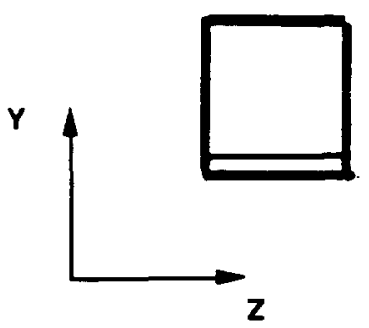

$-15.1 \%$

-Z Recompression

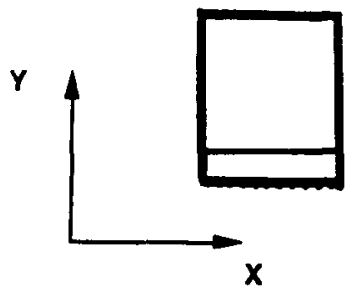

$-13.9 \%$

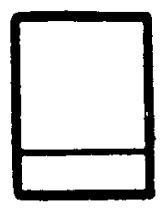

$-16.2 \%$

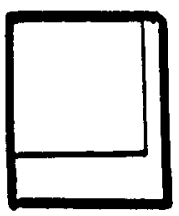

$-43.1 \%$

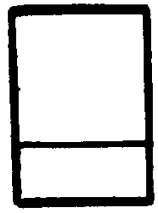

$-27.4 \%$

$-35.7 \%$
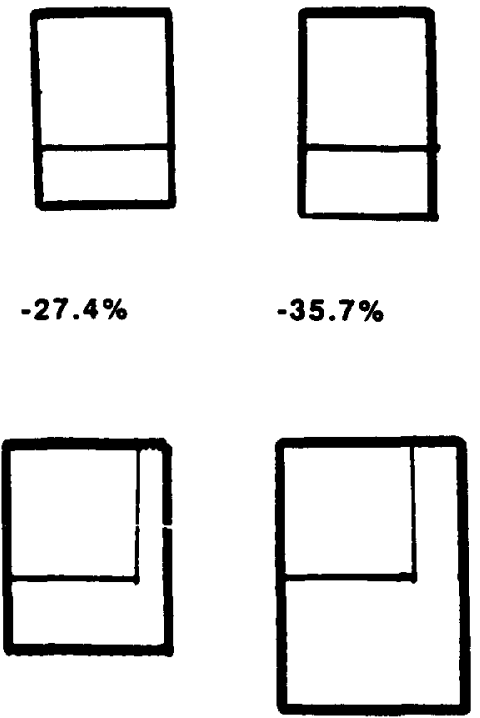

$-61.6 \%$
$-105.0 \%$

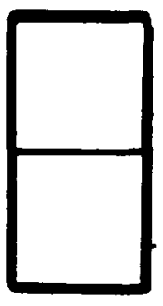

$-67.3 \%$

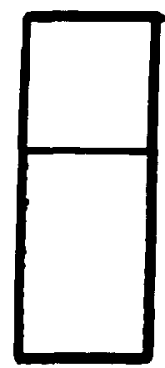

$-89.2 \%$

Fig. 9. Experimentally determined deformation maps from micrometer measurements of the anisotropic evolving cross-section vs. plastic strain in anisotropic PC prestrained in plane-strain compression by $\epsilon=-0.50$ at $\dot{\epsilon}=-0.001 / \mathrm{s}$ and room temperature.

initial orientation prior to reorienting the polymer chains increases the observed extensibility of the anisotropic material during recompression along the $\mathrm{Z}$ direction. The chains in this configuration offer less resistance to the compressive load as they return to a recoiled state; the stress-strain response to this process shows little strain hardening for most of the recompression deformation until the recoiling process is complete and orientation perpendicular to the load axis begins.

The level of uniaxial prestrain is increased to $75 \%$ for the anisotropic responses of Fig. 4. Recompression normal to the oriented chains is again characterized by an 
enhanced flow stress, more rapid strain hardening, and greatly reduced extensibility compared to the isotropic response. As the level of prestrain is increased from $57 \%$ in Fig. 3 to $75 \%$ in Fig. 4, the flow stress is increased and the extensibility is reduced for loading along the $Y$ axis because the increased $Y$ prestrain has increased chain orientation in the $\mathrm{XZ}$ plane. For the anisotropic response along a direction of prior chain alignment seen in Fig. 4, the flow stress is reduced, the strain hardening is delayed and the extensibility is enhanced as compared to the isotropic response; the increase in prestrain has increased the magnitude of each of these effects in the $Z$ direction, which can be seen from a comparison of Figs. 3 and 4.

The uniaxial recompression along the $\mathrm{Z}$ direction of a specimen uniaxially preoriented to a current plastic strain of $51 \%$ was performed by incrementally loading the specimen. After each loading and unloading cycle the dimensions of the specimen were measured with a micrometer giving information on the current level of recompressive strain and the cross-sectional dimensions. The evolving cross-section, which was initially square, has been drawn as a function of recompressive strain in the deformation maps of Fig. 5 . The highly anisotropic flow pattern of this material is further evidence of the effects of preorientation on the glassy polymer response. The $\mathrm{X}$ direction in Fig. 5, for which very little deformation occurs, is a direction of prior chain orientation, whereas the direction of primary material flow (the $\mathrm{Y}$ direction) is the original compression direction. Obviously chain orientation serves as a strong barrier to continued plastic deformation. The material flows very freely along the initial compression direction, and although it is not externally constrained from doing so, material flow is restricted in the oriented direction. The $Y$ direction offers a path of low resistance for chains seeking to stretch and orient in the plane of the cross-section. The previous discussion on the anisotropic strain hardening characteristics described the compression of oriented chains as an easy process when compared to either compressing the isotropic material or compressing the anisotropic material normal to a plane in which chains are oriented. The deformation maps illustrate that it is the combination of compressing oriented chains in the direction of loading and stretching of chains in a direction of low resistance normal to the compression axis that accounts for the very soft response of the anisotropic material in the $\mathrm{Z}$ direction.

Figure 6 contains the results of recompression on a specimen possessing axisymmetric orientation as was the case for the specimens tested in Figs. 3 to 5. In the current case of Fig. 6 the recompression specimen was machined at $45^{\circ}$ to the plane of transverse isotropy, and therefore recompressed at $45^{\circ}$ to the original compression axis. The isotropic response is included in Fig. 6 for comparison. The response of the material to a very high barrier to continued chain alignment at $45^{\circ}$ to the compression axis included extensive specimen shear. This experiment was repeated for the purpose of measuring the deforming specimen cross-section during the recompression. The current height and thickness were determined by micrometer measurement and recorded as a function of plastic strain. The width was calculated assuming incompressibility. ${ }^{3}$

IV.1.2. Initial three dimensional orientation. Results of uniaxial recompression on specimens preoriented to $35 \%$ strain in plane-strain compression appear in Fig. 7 with the isotropic response included for comparison. Plane-strain deformation produces a

\footnotetext{
${ }^{3}$ The size of the preoriented specimen prior to recompression was $2.15 \mathrm{~mm} \times 5.20 \mathrm{~mm} \times 4.55 \mathrm{~mm}$. No accurate measure of the angle of shear during the recompression deformation could be obtained for this size specimen.
} 
fully three dimensional anisotropic state as confirmed by birefringence measurements that yielded different levels of optical anisotropy in the three planes of principal stretch. The amount of plane-strain prestrain is increased to $50 \%$ in the recompression responses of Fig. 8. The three dimensional anisotropy of the plane-strain orientation process is evident from the very different responses of the material in three different directions. The effects of initial anisotropy, seen for the uniaxial preorientation results in Figs. 3 and 4, accumulate more rapidly for preorientation in plane-strain compression because plane-strain deformation provides a one dimensional path for chain extension. Bearing in mind that the anisotropic responses are all due to uniaxial recompression, it is of interest to compare the responses in Figs. 7 and 8 to the results of Figs. 3 and 4, the variables being magnitude of prestrain and state of prestrain. The strain hardening characteristics for recompression after plane-strain preorientation all occur in response to a nonaxisymmetric barrier to continued plastic flow. Each such recompression offers a preferential path for material flow; the rate at which strain hardening evolves is dependent on the strength of the weaker barrier, with the result of the weakest combination of barriers yielding the softest response.

The deformation maps in Fig. 9 further illustrate the effects of an anisotropic barrier to plastic flow. The evolving cross-section dimensions have been plotted vs. recompressive plastic strain in Fig. 9 for all three orientations of recompression specimens prestrained to $50 \%$ in plane strain. Because all three cross-sections are in no way externally impeded from expanding, the effects of the anisotropic resistance to plastic flow are clearly seen to be responsible for the direction-dependent flow patterns presented. The degree of the existing anisotropy in the plane of the cross-section is directly apparent from the deformed shape of the cross-section. The magnitudes of the individual barriers to plastic flow can be inferred by comparison of deformation maps and corresponding stress-strain curves. The material is strongest in recompression along the $Y$ direction for which continued plastic flow requires the further orientation of chains in two previously oriented directions. Extensibility is greatly reduced in this direction owing to the existing chain orientation. These results are similar to those found for the transversely isotropic material in that the response of the anisotropic material is a function of the three dimensional nature of anisotropy, depending on the level of chain orientation both in the direction of loading and in the plane normal to the compressive load.

The anisotropic effects illustrated here have implications for both the processing of solid polymer components from anisotropic preforms and the predicting of the structural integrity of anisotropic polymer components. Materials containing anisotropy can have very different responses from those expected even if the anisotropy is not extensive. The initial yield response is greatly changing during strain softening, before any appreciable anisotropy develops due to chain orientation.

IV.1.3. Effects of thermal and strain history. The ability to characterize the orientation induced mechanical anisotropy in glassy polymers by the measurement of optical anisotropy was investigated by the results of Fig. 10 for two PMMA specimens subjected to different strain and thermal histories. Both specimens were preoriented in uniaxial compression; the temperature during deformation and magnitude of prestrain were variables. The stress-strain responses of two tests, one conducted at $23^{\circ} \mathrm{C}$ to a final strain of $89 \%$, the other tested at $75^{\circ} \mathrm{C}$ to $104 \%$ strain, are presented in Fig. 10A. Upon unloading, and also cooling in the case of the $75^{\circ} \mathrm{C}$ test, the materials recovered to $66 \%$ and $83 \%$, respectively, these strains are marked on Fig. $10 \mathrm{~A}$ and represent the strains 

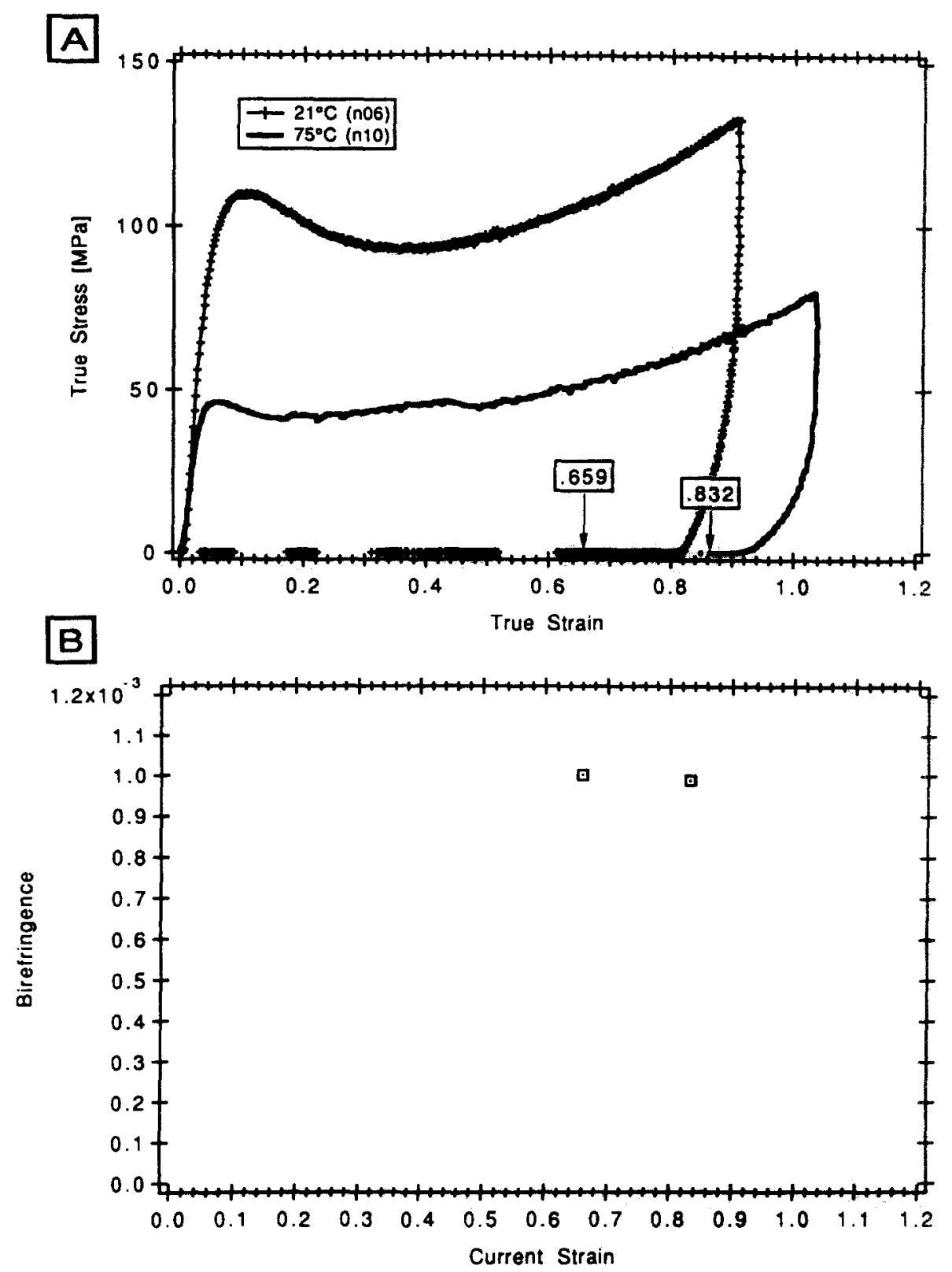

Fig. 10. Experimentally determined uniaxial compression stress-strain curves of isotropic PMMA under different thermal and strain histories; one tested at room temperature to a final strain under load of $\epsilon=-0.89$ at $\dot{\epsilon}=-0.001 / \mathrm{s}$, the other subjected to a strain of $\epsilon=-1.04$ at $\dot{\epsilon}=-0.001 / \mathrm{s}$ and $75^{\circ} \mathrm{C}$. (B) Birefringence vs. strain for the specimens tested in (A) confirming that the specimens contain the same level of anisotropy as measured by birefringence.

in the materials at the time of recompression. The two tests obviously represent two different strain and thermal histories. Residual birefringence measurements made on the specimens after recovery verify that these two specimens contain the same level of optical anisotropy. The birefringence vs. current plastic strain results for these specimens are given in Fig. 10B. 
The preoriented materials were machined to supply two anisotropic specimens each for retesting under identical conditions at $23^{\circ} \mathrm{C}$ both parallel and perpendicular to the plane of chain orientation. Results of these recompression tests are given in Fig. 11 and show that the highly anisotropic response of these materials, characterized by flow strength, strain hardening slopes, and limiting extensibilities, are remarkably similar. The material property common to each of these specimens is the optical anisotropy measured by birefringence. Characterization of anisotropy in terms of the measure of birefringence shows great promise as a predictor of highly anisotropic mechanical behavior because of the similarity of results in Fig. 11. The authors submit this experimental result as evidence for the use of optical anisotropy as a state variable, indicative of the three dimensional orientation state of polymers, and capable of a measurable amount of evolution with temperature as well as magnitude of strain and state of strain as previously documented in ARRUDA and BOYCE [in press]. Knowledge of the prior strain or thermal history of an anisotropic polymer preform or component would not be necessary for predicting its response to a processing operation or service loads, provided the effects of anisotropy such as direction-dependent flow strengths, strain hardening characteristics, limiting extensibilities, and deformation patterns presented here are well characterized. In the section that follows, the successful characterization of the isotropic state in terms of the three dimensional backstress tensor is shown to fully predict the anisotropic behavior. A basis is established by this work for incorporating the measurable evolution of birefringence into the existing framework of a model for an evolving molecular network.

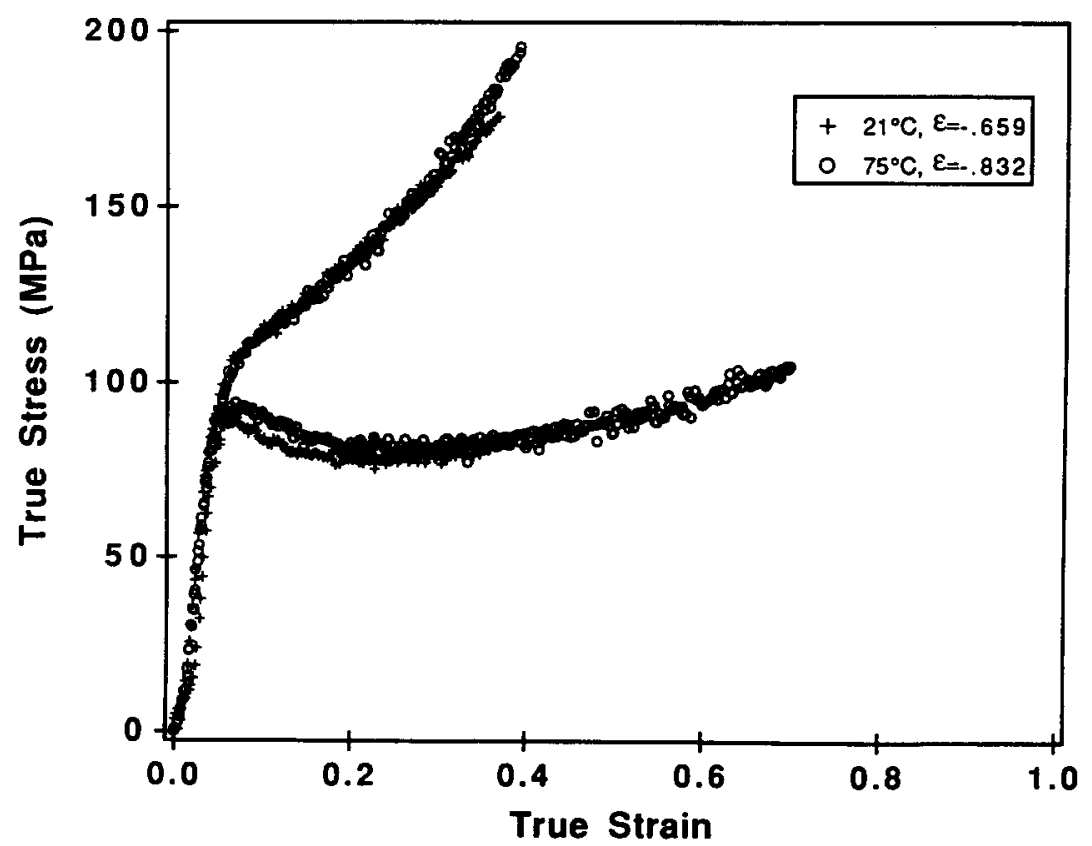

Fig. 11. Experimentally determined uniaxial compression stress-strain curves of the anisotropic response of PMMA as a result of transverse isotropy in materials subjected to the different thermal and strain histories shown in Fig. 10A; one prestrained by $\epsilon=-0.66$ at $\dot{\epsilon}=-0.001 / \mathrm{s}$ and room temperature, the other prestrained by $\epsilon=-0.83$ at $\dot{\epsilon}=-0.001 / \mathrm{s}$ and $75^{\circ} \mathrm{C}$. 


\section{IV.2. Comparisons with simulations}

The procedure used to characterize the evolution of anisotropy during large strain deformation of glassy polymers has been described in detail in ARRUDA and BOYCE [in press] and will only be summarized here. The rate and temperature dependence of initial yield, described by the Argon expression in eqn 2, requires two tests to characterize the rate and temperature dependencies of the material. Characterization of the strain hardening response requires one test to determine the two physically based parameters of the tensor resistance in eqn 16. One of the tests required for the rate- and temperature-dependent characteristics serves also as the test required for obtaining the additional information on strain hardening, thus the total number of required tests is two. The properties required of the strain hardening response are the limiting chain extensibility, $\sqrt{N}$, and the rubbery modulus, $C_{R}=n k \theta$, these are determined by fitting values to the uniaxial compression curve shown in Fig. 2. Characterization of the evolution of anisotropy in the initially isotropic polymer is complete for isothermal responses with this process. The large strain response of the isotropic polymer to any state of deformation can be predicted based on the characterization of the original uniaxial compression response without changing the material properties determined from the uniaxial compression response. The plane-strain compression response of $\mathrm{PC}$ is simulated in this manner.

The results in Fig. 12 illustrate the ability of the model to predict the strong state of deformation dependence of glassy polymers as discussed in ARRUDA and BOYCE [in

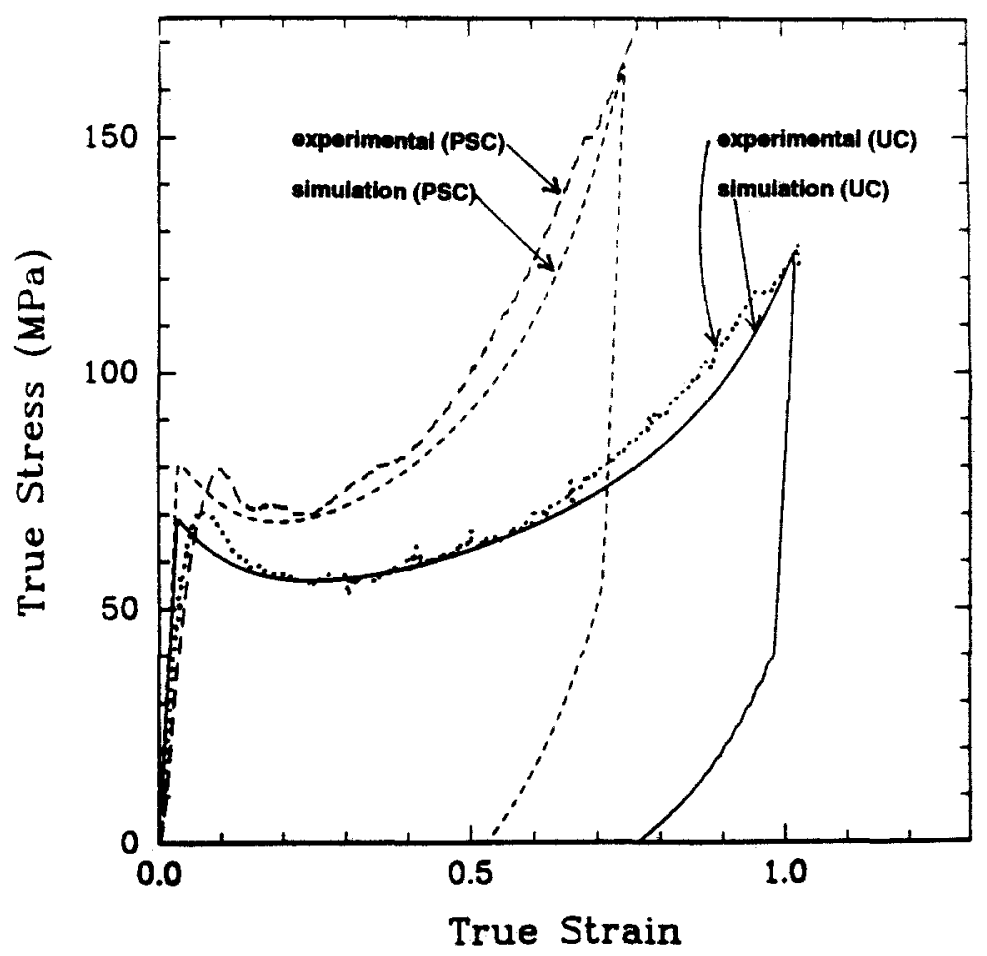

Fig. 12. Eight chain model simulations and experimental curves of the uniaxial compression and plane-strain compression responses of $\mathrm{PC}$ at $\dot{\epsilon}=-0.001 / \mathrm{s}$. The model was fitted to the uniaxial response, then the planestrain response was predicted based on uniaxial properties. 
press]. The model has been fit to the uniaxial compression data in Fig. 12 and used to predict the plane-strain compression data, also in the figure. The current theory models the initial response of the glassy polymer as linear elastic to the point of the yield stress. Yield is itself modelled as an instantaneous process, resulting in immediate strain softening of the simulated response. In contrast, the actual material undergoes a viscoplastic response after approximately $-0.05 \%$ strain, and the actual yield event is the result of a thermally activated and rate-sensitive number of transformations that occur over a range of straining, evidence of the actual material response has been given in HASAN, BOYCE, LI, and Berko [1993]. The simulations of the anisotropic responses continue to predict linear elastic behavior followed by a sudden imposition of inelastic deformation, whereas the experimental results show a more gradual transition to inelastic deformation.
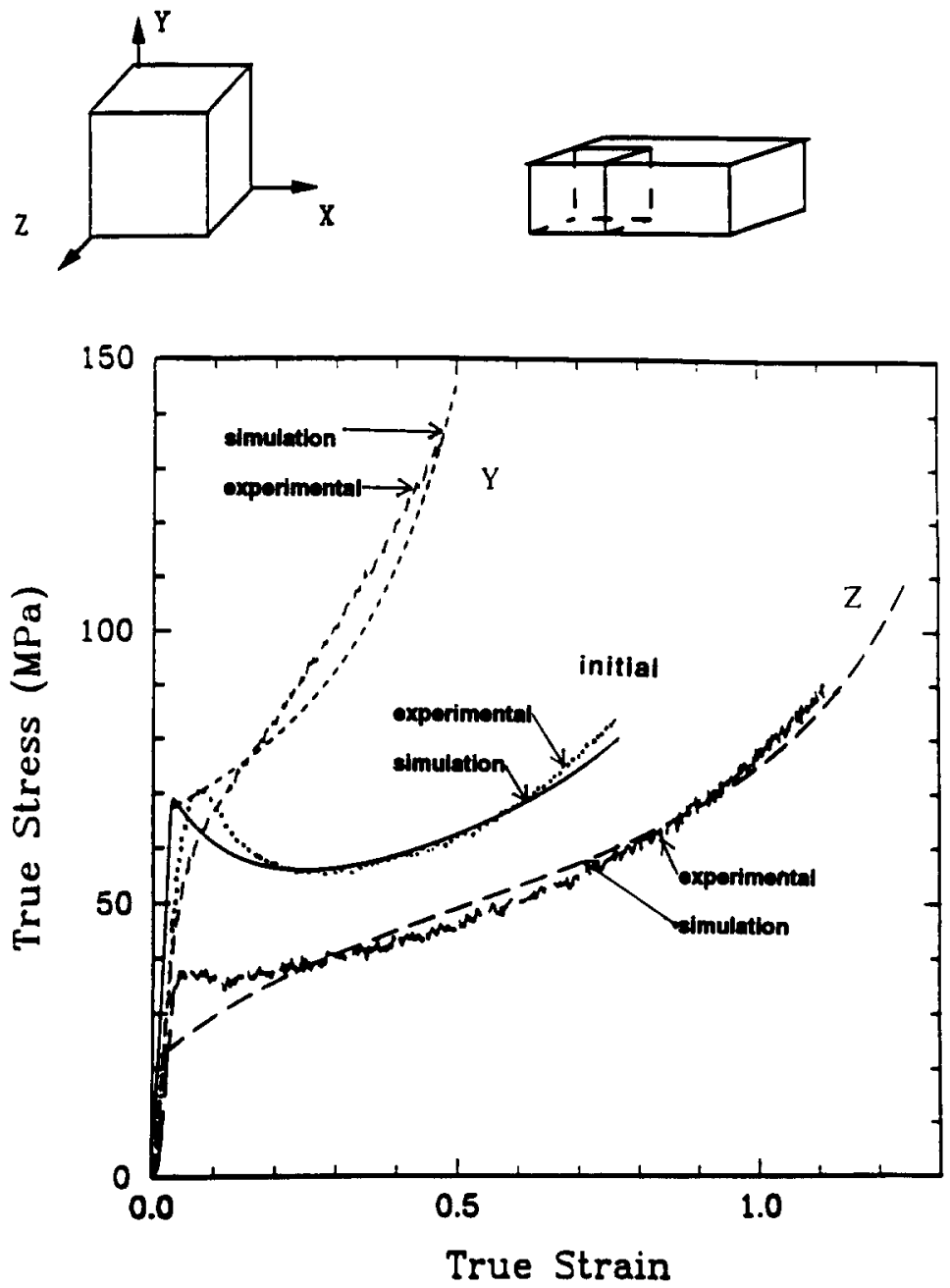

Fig. 13. Comparison of theory with experiments described in Fig. 3 for the anisotropic response of transversely isotropic PC prestrained by $\epsilon=-0.57$ at $\dot{\epsilon}=-0.001 / \mathrm{s}$ and room temperature. 
IV.2.1. Predicting the effects of initial axisymmetric anisotropy. The incorporation of the initial orientation into the model is achieved in terms of an initial deformation gradient, $\mathbf{F}^{i}$, which produces an initial backstress tensor, as described in Section III.3. No additional parameters are introduced to simulate the anisotropic effects presented earlier. Complete characterization of the evolving backstress tensor is achieved by the single uniaxial compression test in Fig. 12 and the knowledge of the anisotropic state of the material described by $\mathbf{F}^{i}$.

The comparison of theory with experiment begins with the anisotropic response of a material preoriented to $57 \%$ strain in uniaxial compression, which was described in Fig. 3, and is reproduced along with the results of the simulations in Fig. 13. The strongly direction-dependent responses of preoriented PC are very accurately predicted with the model. The theory captures the anisotropic flow stresses, the strain hardening behaviors, and the limiting extensibilities as they have been described. In particular, the
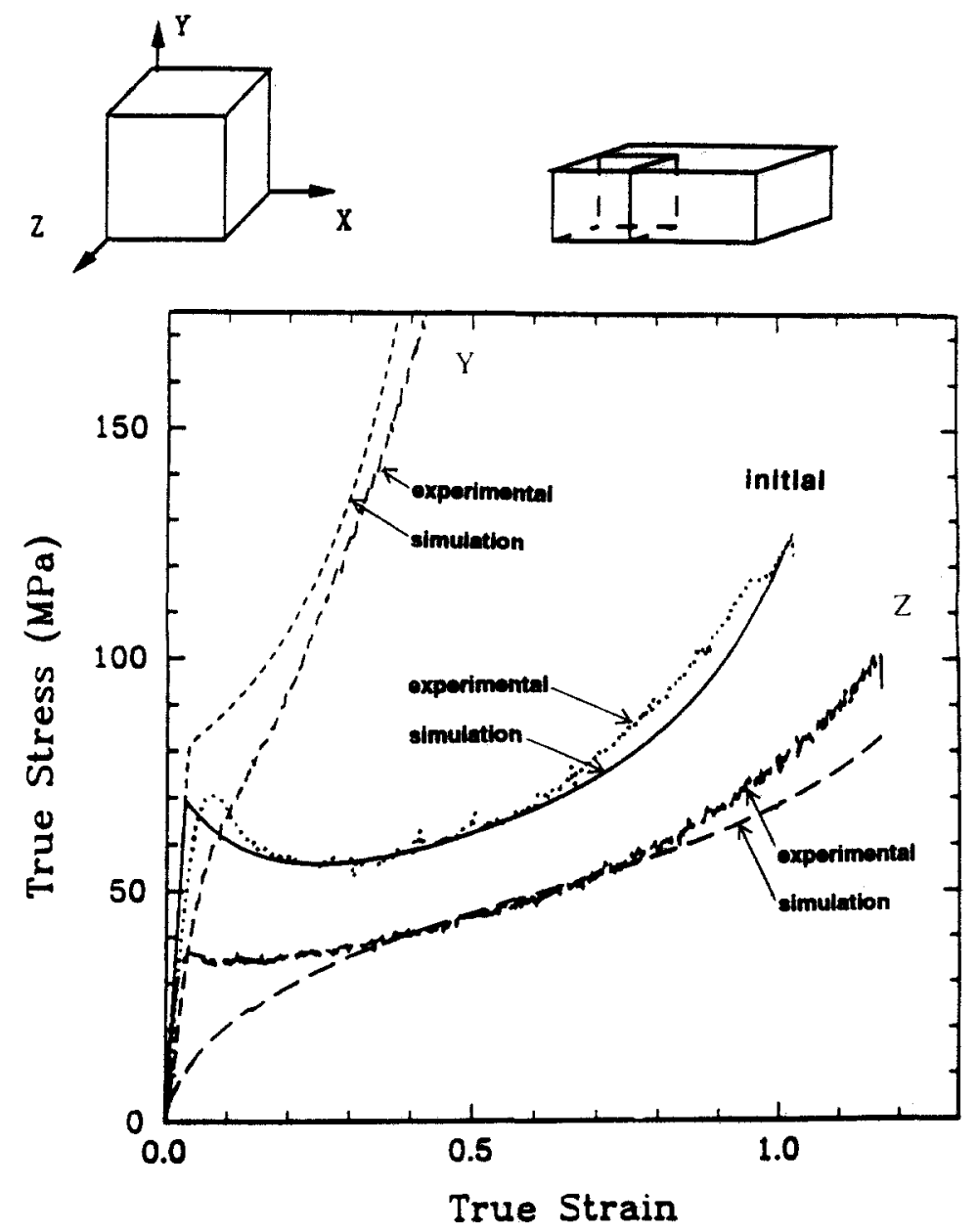

Fig. 14. Comparison of theory with experiments described in Fig. 4 for the anisotropic response of transversely isotropic PC prestrained by $\epsilon=-0.75$ at $\dot{\epsilon}=-0.001 / \mathrm{s}$ and room temperature. 
simulation of the recompression along the $Z$ axis predicts the extensive softening effect owing to the compression of coiled chains during this deformation. The simulation matches the highly anisotropic experimental result for the entire recompressive strain, capturing the enhanced extensibility of the material in this direction. The anisotropic response along the $\mathrm{Y}$ axis appears in stark contrast to the $\mathrm{Z}$ direction recompression. The true usefulness of the theory is its ability to very accurately characterize this highly anisotropic behavior, capturing the rapid accumulation of orientation-induced strain hardening that leads to reduced extensibility in this direction.

Increasing the level of prestrain in the anisotropic material was shown earlier to intensify the differences in the direction-dependent responses. As the results in Fig. 14 show, the model continues to capture the effects of initial anisotropy as the level of anisotropy increases.

The size of the deforming anisotropic cross-section was monitored during the compression simulation. The predicted deformation map has been plotted versus recompressive strain in Fig. 15 for comparison with Fig. 5. Agreement between theory and experiment is extremely good; the deforming cross-section simulation results from an accurate characterization of the relative strengths of the barriers to plastic flow that exists in the cross-section. The simulations predict that virtually no plastic flow occurs along the $\mathrm{X}$ direction after $-97.5 \%$ recompressive plastic strain, a remarkable testimony to the relative strength of the barrier to continued plastic flow along the $\mathrm{X}$ axis, which is the result of highly oriented chains in this direction.

Fig. 16 contains the comparison of theory with experiment for the transversely isotropic material recompressed along the $Y^{\prime}$ axis such that the plane of principal chain orientation was at $45^{\circ}$ to the recompression axis. The figure shows how remarkably well the theory predicts the stress-strain response of the specimen that undergoes extensive shear as part of its anisotropic response to the recompression. The strain hardening

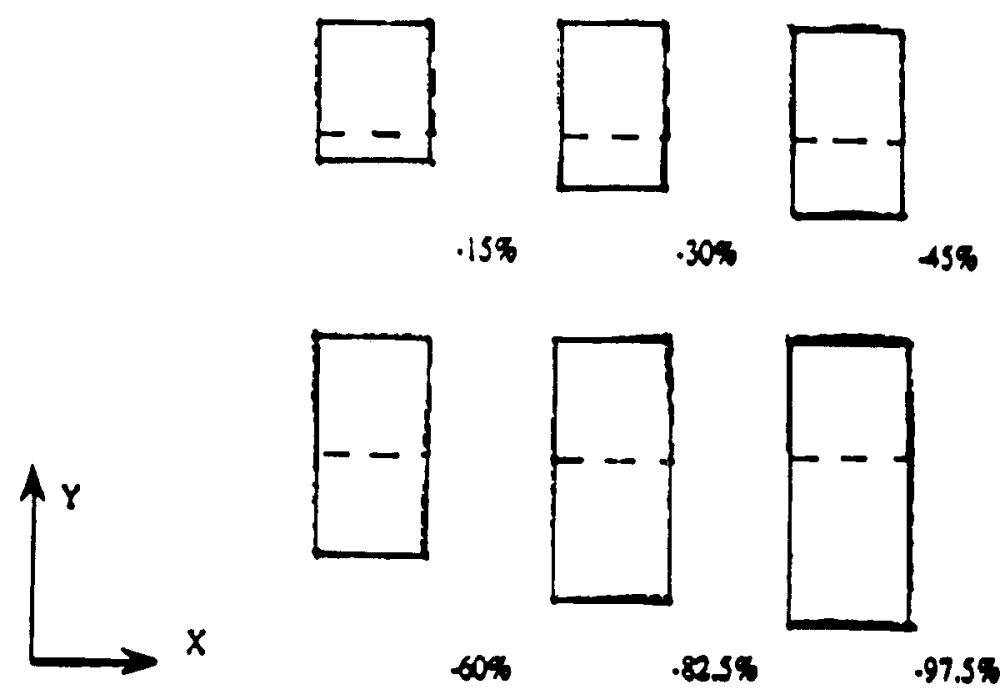

Fig. 15. Simulations of the anisotropic response of the evolving cross-section during recompression vs. plastic strain in anisotropic PC prestrained in uniaxial compression by $\epsilon=-0.51$ at $\dot{\epsilon}=-0.001 / \mathrm{s}$ and room temperature. Compare with experiments described in Fig. 5. 

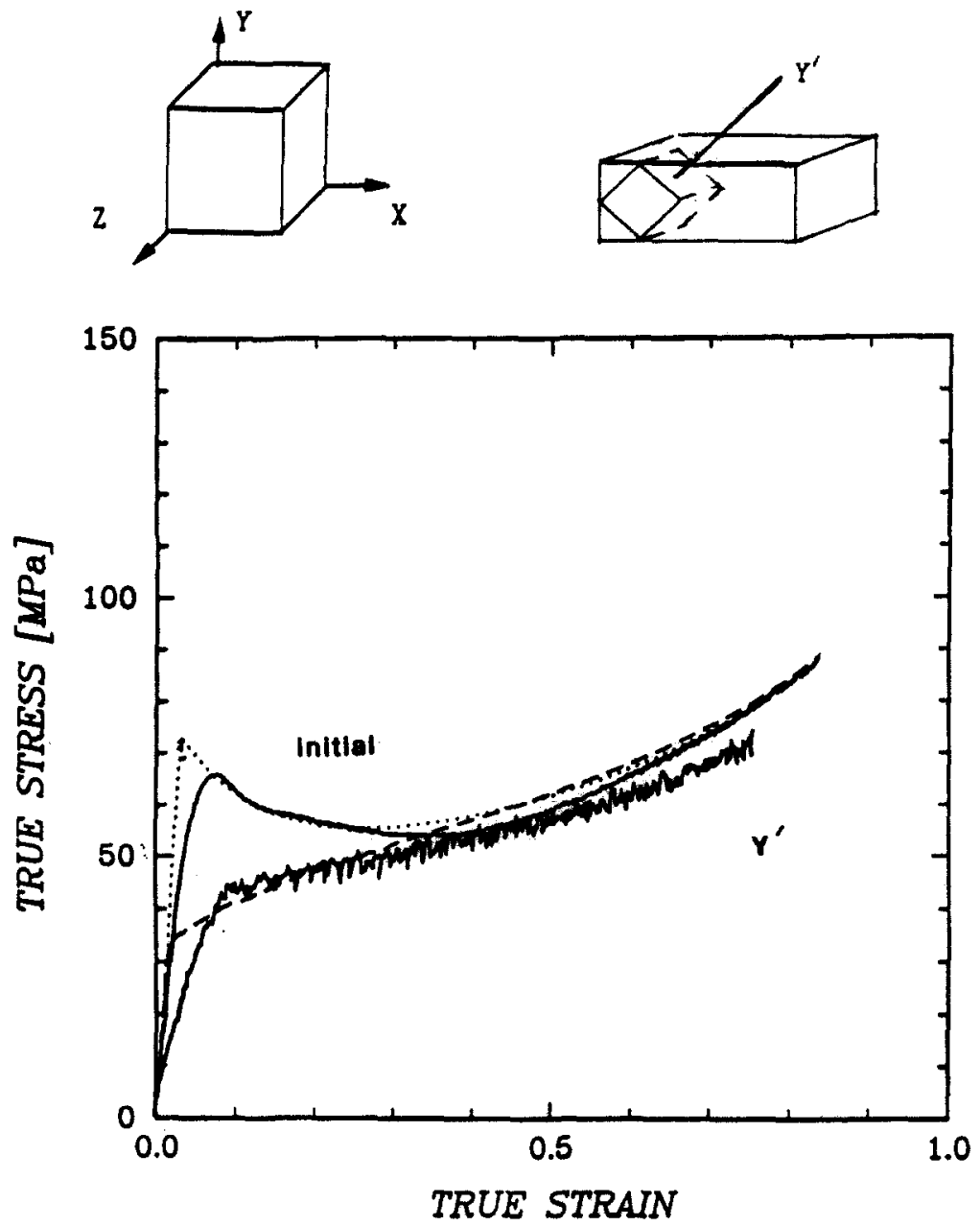

Fig. 16. Comparison of theory with experiments described in Fig. 6 for the anisotropic response of PC prestrained by $\epsilon=-0.75$ at $\dot{\epsilon}=-0.001 / \mathrm{s}$ and room temperature. The plane of principal chain orientation is oriented at $45^{\circ}$ to the recompression direction, resulting in extensive specimen shear during the recompression.

response of this specimen is linear to $-60 \%$ strain, at which point an increase in the slope of the stress-strain response can be detected. The theory accurately captures this effect, predicting an initially linear strain hardening response, albeit at a slightly higher slope, then capturing the onset of an increasing strain hardening slope at $60 \%$ strain. The prediction lies within $5 \mathrm{MPa}$ of the actual response throughout the strain hardening portion of the stress-strain curve. The simulated deformation maps actually predict specimen shear during the recompression, but because an accurate measure of the actual experimentally observed specimen shear could not be obtained, the deformation maps cannot be included for comparison. A comparison has been made in Table 2 of the size of the deformed specimen versus current plastic strain as determined from micrometer measurements ${ }^{4}$ and predicted in the simulations.

\footnotetext{
${ }^{4}$ The width dimension was calculated from incompressibility as described in section IV.1.1.
} 
Table 2. Comparison of the experimentally determined and predicted normalized sizes of the cross-section of the deforming specimen described in Figs. 6 and 16 at various levels of recompressive strain

\begin{tabular}{ll}
\hline Experiment & Simulation \\
\hline$\epsilon=-25.9 \%$ & $\epsilon=-28.9 \%$ \\
$t=1.0442$ & $t=1.0440$ \\
$w=1.2395$ & $w=1.2400$ \\
$\epsilon=-55.8 \%$ & $\epsilon=-53.8 \%$ \\
$t=1.1190$ & $t=1.0890$ \\
$w=1.5610$ & $w=1.5670$ \\
$\epsilon=-69.3 \%$ & $\epsilon=-66.4 \%$ \\
$t=1.1760$ & $t=1.1200$ \\
$w=1.7000$ & $w=1.7160$ \\
\hline
\end{tabular}
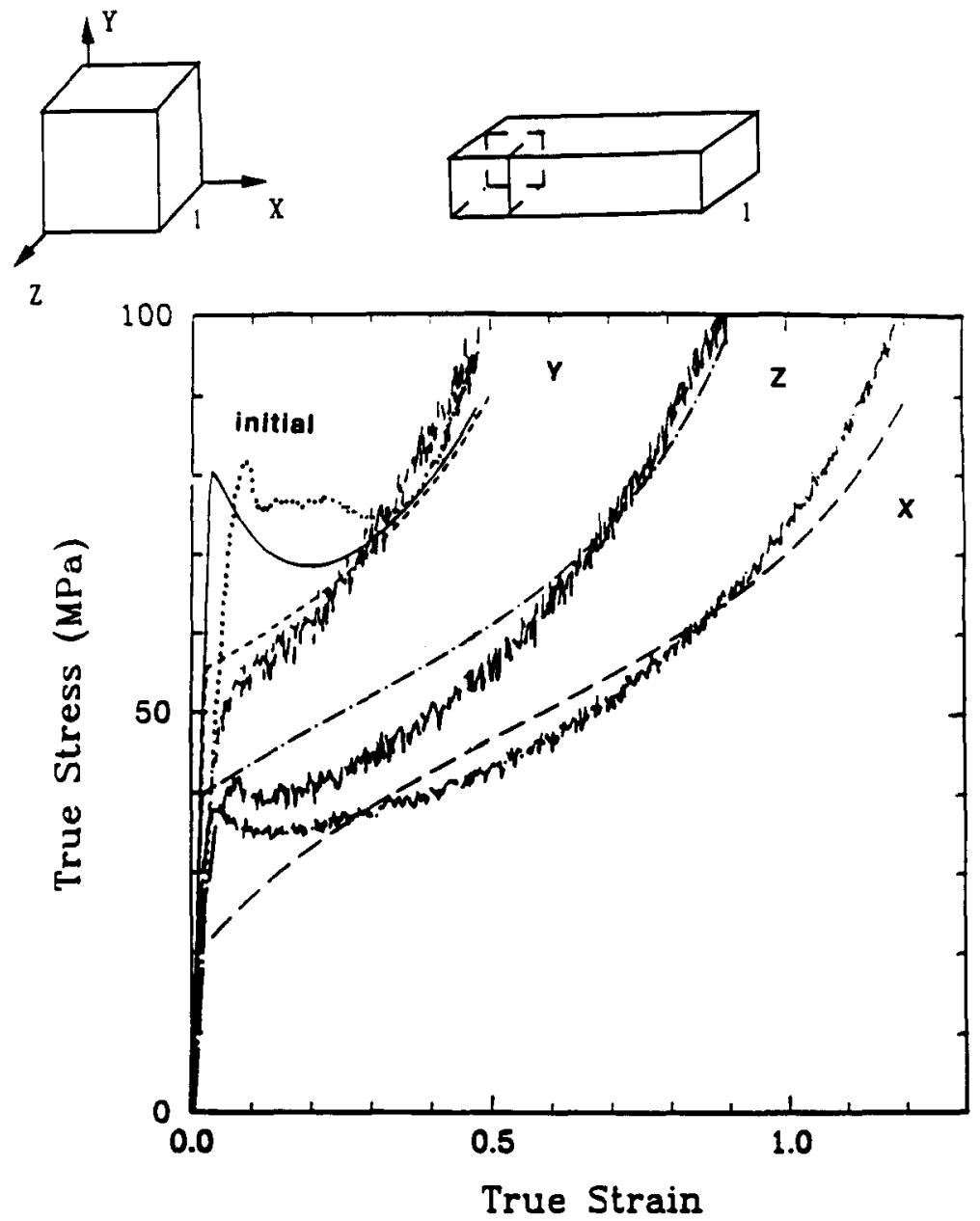

Fig. 17. Comparison of theory with experiments described in Fig. 7 for the anisotropic response of three dimensionally anisotropic PC prestrained by $\epsilon=-0.35$ at $\dot{\epsilon}=-0.001 / \mathrm{s}$ and room temperature. 
IV.2.2. Predicting the effects of initial three dimensional anisotropy. Results of simulations on the anisotropic response of materials containing three dimensional anisotropy as a result of initial deformation in plane-strain compression are shown with the experimental data in Figs. 17 to 19. The stress-strain responses, which vary significantly in all three directions, are again very accurately captured by the model. The simulations required the characterization of the isotropic response in uniaxial compression and the knowledge of the anisotropic state of the material to accurately account for the three dimensional nature of changes in flow stresses, extensibilities, strain hardening characteristics, and deformation patterns. The simulations are particularly accurate in capturing the shapes of the strain hardening curves, matching slopes, and extension limits with the experimental data in all three directions. The predictive capabilities of the theory are perhaps best represented in the three recompression deformation maps of Fig. 19, which

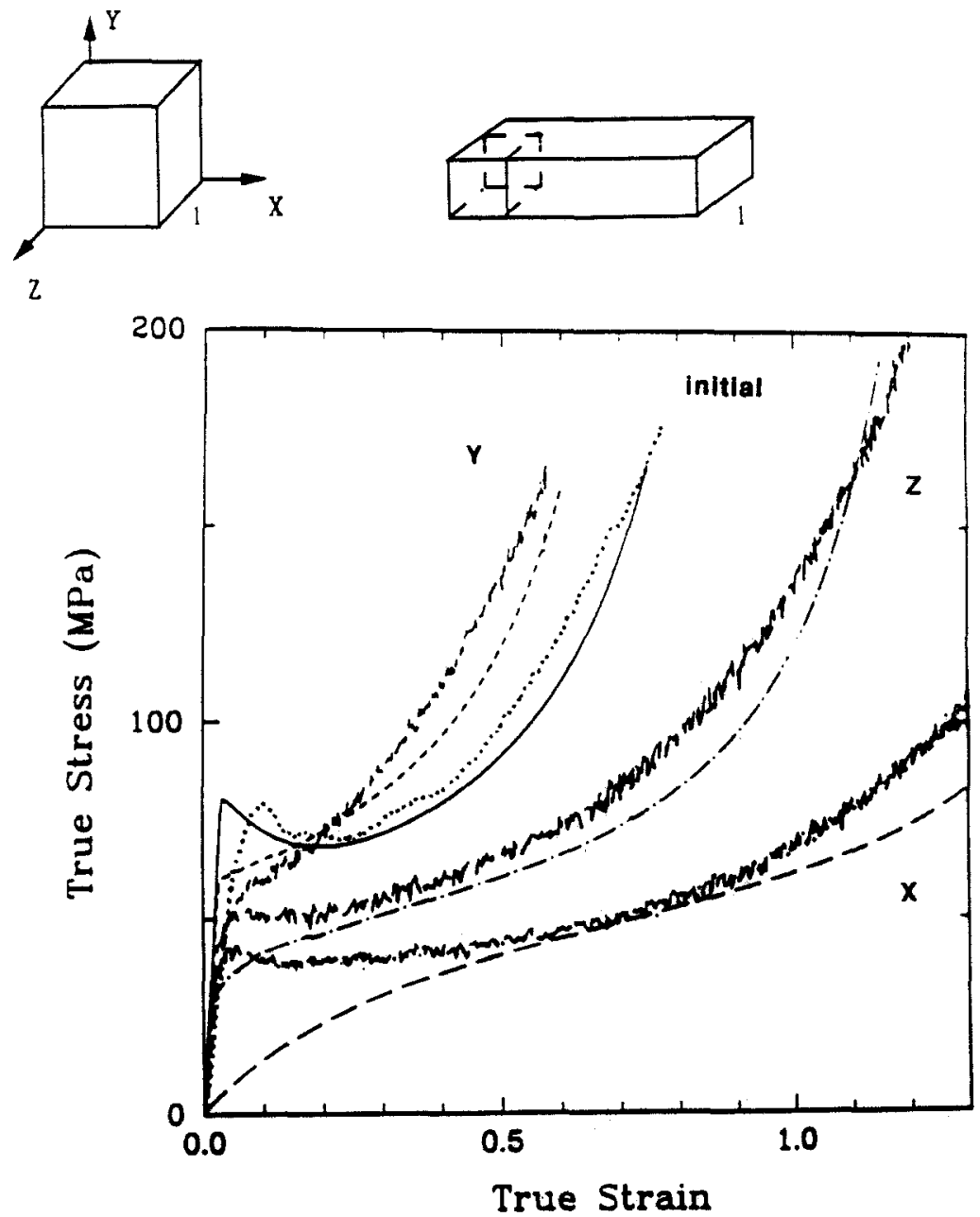

Fig. 18. Comparison of theory with experiments described in Fig. 8 for the anisotropic response of three dimensionally anisotropic PC prestrained by $\epsilon=-0.50$ at $\dot{\epsilon}=-0.001 / \mathrm{s}$ and room temperature. 


\section{-Y Recompression}

$\mathbf{z}$

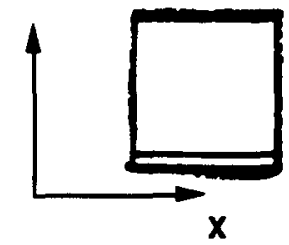

$-6.2 \%$

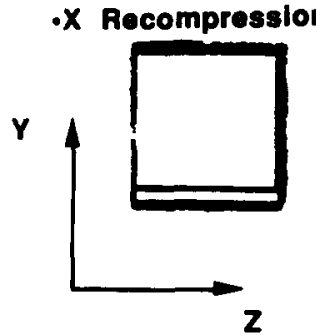

$-15.1 \%$

.z Recompression

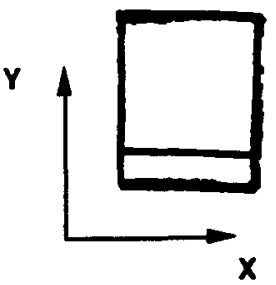

$-13.9 \%$

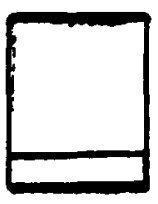

$-16.2 \%$

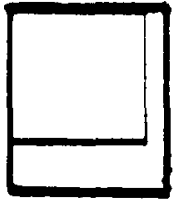

$-43.1 \%$

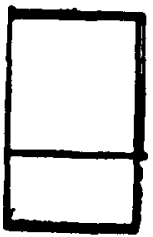

$-32.8 \%$

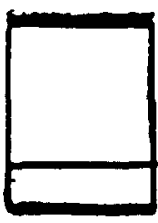

$-27.4 \%$

$-35.7 \%$
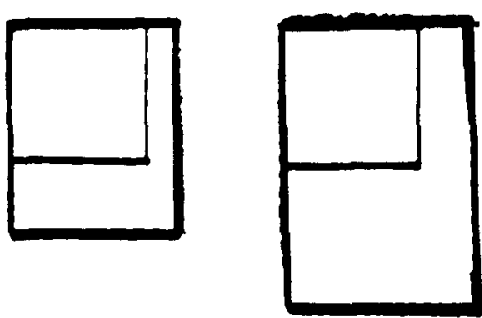

$-105.0 \%$

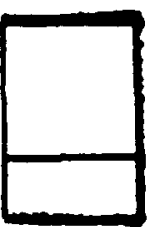

$-61.6 \%$
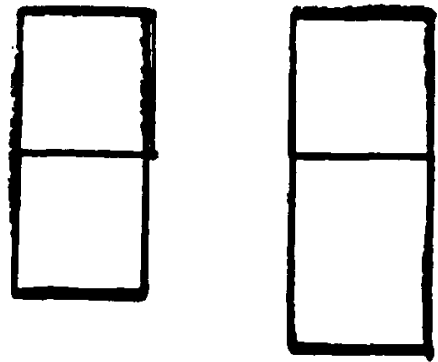

$-89.2 \%$

Fig. 19. Simulations of the anisotropic response of the evolving cross section during recompression vs. plastic strain in anisotropic PC prestrained in plane-strain compression by $\epsilon=-0.50$ at $\dot{\epsilon}=-0.001 / \mathrm{s}$ and room temperature. Compare with experiments described in Fig. 9.

are almost exact replicas of the experimentally determined counterparts in Fig. 9. The relative strengths of the barriers to continued plastic flow in directions $\mathrm{X}, \mathrm{Y}$, and $\mathrm{Z}$ can be directly determined from these deformation maps. The implications these results have for the problems of processing anisotropic polymers and using processed polymer components in structural applications are astounding. We are unaware of any other models or studies that can predict such a wide range of anisotropic behaviors for the full range of material extensibility as in this study. Clearly the isothermal response of these materials has been fully and accurately described by the model developed in ARRUDA and BOYCE [in press] and verified here. 


\section{SUMMARY}

The effects of initial anisotropy have been experimentally observed to include direction-dependent flow stresses, strain hardening characteristics, limiting extensibilities, and deformation patterns. The existing anisotropy is a strong function of the deformation state used to preorient the material. Two anisotropic states were examined here, the transversely isotropic state developed during preorientation in uniaxial compression and the fully three dimensional state of anisotropy produced during plane-strain deformation. The remarkably direction-dependent responses of anisotropic glassy polymers warrant thorough characterization for applications involving processing of anisotropic materials or the use of anisotropic polymer components in service.

A model developed for the evolution of anisotropy during large strain deformations in glassy polymers in ARRUDA and BOYCE [in press] was used here to predict the anisotropic effects in preoriented materials. The model requires one test to characterize the evolving anisotropy and the knowledge of the current state of anisotropy in the preoriented materials. All aspects of the anisotropy were well predicted with the model confirming for the first time in any material the ability to completely capture the essence of three dimensional anisotropy under large strain deformations.

An experimental observation that the anisotropic response of glassy polymers can be characterized by the current level and three dimensional nature of anisotropy, regardless of the strain or thermal history that produced the anisotropy, was included. Birefringence has been targeted as a basis for directly incorporating the material characterization of anisotropy into the anisotropic backstress element of the existing model.

Acknowledgements - This work is sponsored by The National Science Foundation (MSM-8818233) and the MIT Bradley Foundation.

\section{REFERENCES}

1950 Hill, R., The Mathematical Theory of Plasticity, Oxford University Press, Oxford.

1968 Brown, N., Duckett, R.A., and WARD, I.M., “The Yield Behavior of Oriented PET," Philosophical Magazine, 18, 483.

1968 Brown, N., and WARD, I.M., "Deformation Bands in Oriented PET," Philosophical Magazine, 17, 961.

1969 LeE, E.H., "Elastic-Plastic Deformation at Finite Strain," ASME Journal of Applied Mechanics, $56,512$.

1969 Rider, J.G., and Hargreaves, E., "Yielding of Oriented Polyvinylchloride," Journal of Polymer Science, A-2, 7, 829.

c1970 Graham, M.D., "Retardation in $n m$, for Plate Angle, $i$, in Degrees, for Berek Compensator S-N 3311," (Tech. rep.), Massachusetts Institute of Technology, Cambridge, MA.

1971 Rawson, F.F., and RIDER, J.G., "Effects of Internal Stress on the Yielding of Oriented PVC," Journal of Polymer Science B, 26, 87 .

1973 Argon, A.S., "A Theory for the Low-Temperature Plastic Deformation of Glassy Polymers," Philosophical Magazine, 28, 839.

1979 ANAND, L., "On H. Hencky's Approximate Strain Energy Function for Modelling Deformations," ASME Journal of Applied Mechanics, 46, 78.

1984 WARD, I.M., "The Role of Molecular Networks and Thermally Activated Processes in the Deformation Behavior of Polymers," Polymer Engineering and Science, 24, 724.

1986 LEe, D., and Luken, P.C., "Material Modelling and Solid Phase Forming of Polycarbonate Sheet," Polymer Engineering and Science, 26, 612.

1987 Vest, T.A., Amoedo, J., and LeE, D., "Modelling of Tensile Stress Behavior in Semi-Crystalline and Amorphous Polymers," ASME Publication AMD-V85, 71.

1988 ADAMS, G.W., and FARRIS, R.J., "Latent Energy of Deformation of Bisphenol A Polycarbonate," Journal of Polymer Science, 26, 433.

1988 Boyce, M.C., Parks, D.M., and Argon, A.S., "Large Inelastic Deformation of Glassy Polymers, Part I: Rate Dependent Constitutive Model," Mechanics of Materials, 7, 15. 
1989 BERG, E.M., SUN, D.C., and MAGLI, J.H., "3-D Structure-Property Relationships in Rolltruded Polymers, Part I: Mechanical Property Enhancement in Three Directions," Polymer Engineering and Science, 29, 715 .

1989 BoyCe, M.C., Parks, D.M., and ARgon, A.S., "Plastic Flow in Oriented Polymers," International Journal of Plasticity, 5, 593.

1989 Boyce, M.C., Weber, G.G., and Parks, D.M., "On the Kinematics of Finite Strain Plasticity," Journal of the Mechanics and Physics of Solids, 37, 647.

1990 OleynIK, E., In Baer, E., and Moet, S., (eds.), High Performance Polymers, Hauser, Munich, p. 79.

1993 ARRUdA, E.M., and BoYCE, M.C., "A Three-Dimensional Constitutive Model for the Large Stretch Behavior of Rubber Elastic Materials," Journal of the Mechanics and Physics of Solids, 41, 389.

1993 Hasan, O.A., Boyce, M.C., Li, X.S., and Berko, S., "An Investigation of the Yield and Post-Yield Behavior and Corresponding Structure of Glassy Polymers," Journal of Polymer Science, Part B: Polymer Physics Edition, 31, 185.

In press BoyCE, M.C., ARruda, E.M., and Jayachandran, R., "Strain Rate and Temperature Effects on the Inelastic Behavior of Glassy Polymers."

In press ARRUDA, E.M., and BoyCE, M.C., "Evolution of Plastic Anisotropy in Amorphous Polymers During Finite Straining," International Journal of Plasticity.

Department of Mechanical Engineering

and Applied Mechanics

University of Michigan

Ann Arbor, MI 48109, USA

(Received 6 April 1992; in final revised form 14 April 1993) 